\title{
Stochastic Burgers Type Equations with Reflection: Existence, Uniqueness
}

DOI:

10.1016/j.jde.2019.05.008

\section{Document Version}

Accepted author manuscript

Link to publication record in Manchester Research Explorer

\section{Citation for published version (APA):}

Zhang, T. (2019). Stochastic Burgers Type Equations with Reflection: Existence, Uniqueness. Journal of Differential Equations. https://doi.org/10.1016/j.jde.2019.05.008

\section{Published in:}

Journal of Differential Equations

\section{Citing this paper}

Please note that where the full-text provided on Manchester Research Explorer is the Author Accepted Manuscript or Proof version this may differ from the final Published version. If citing, it is advised that you check and use the publisher's definitive version.

\section{General rights}

Copyright and moral rights for the publications made accessible in the Research Explorer are retained by the authors and/or other copyright owners and it is a condition of accessing publications that users recognise and abide by the legal requirements associated with these rights.

\section{Takedown policy}

If you believe that this document breaches copyright please refer to the University of Manchester's Takedown Procedures [http://man.ac.uk/04Y6Bo] or contact uml.scholarlycommunications@manchester.ac.uk providing relevant details, so we can investigate your claim.

\section{OPEN ACCESS}




\title{
Stochastic Burgers Type Equations with Reflection: Existence, Uniqueness
}

\author{
Tusheng Zhang ${ }^{1}$
}

November 23, 2018

\begin{abstract}
In this paper, we establish the existence and uniqueness of solutions of reflected stochastic Burgers-type equations. The main difficulty is to deal with the highly non-linear coefficients and the singularities caused by the reflection.
\end{abstract}

Keywords: Stochastic Burgers-type equations; Stochastic partial differential equations with reflection; Random measures; Sobolev embedding.

AMS Subject Classification: Primary 60H15 Secondary 60J60, 35R60.

\section{Introduction and framework}

Consider the following Burgers-type stochastic partial differential equation (SPDE) with reflection:

$$
\begin{aligned}
d u(t, x) & =\frac{\partial^{2} u(t, x)}{\partial x^{2}} d t+\frac{\partial g(u(t, x))}{\partial x} d t+\sum_{j=1}^{m} \sigma_{j}(u(t, x)) d B_{j}(t)+d L(t, x), \quad x \in[0,1], \\
u(t, x) & \geq 0 \\
u(0, \cdot) & =u_{0}(\cdot) \geq 0, \quad u(t, 0)=u(t, 1)=0,
\end{aligned}
$$

where $B=\left(B_{1}, B_{2}, \ldots B_{m}\right)$ denotes a $m$-dimensional Brownian motion on a complete probability space $\left(\Omega, \mathcal{F},\left\{\mathcal{F}_{t}\right\}_{t \geq 0}, P\right)$, where $\mathcal{F}_{t}=\sigma(B(s): 0 \leq s \leq t) ; u_{0}$ is a non-negative function on $[0,1]$; $d L(t, x)$ is a random measure which is a part of the solution pair $(u, L)$. The coefficients $g(\cdot)$ and $\sigma(\cdot)=\left(\sigma_{j}(\cdot), j \geq 1\right)$ are non-linear real-valued functions which will be specified later. The following is the definition of a solution.

Definition 1.1. A pair $(u, L)$ is said to be a solution of equation (1.1) if

(i) $u$ is a continuous random field on $\mathbb{R}_{+} \times[0,1] ; u(t, x)$ is $\mathcal{F}_{t}$ measurable and $u(t, x) \geq 0$ a.e.

(ii) $L$ is a random measure on $\mathbb{R}_{+} \times[0,1]$ such that

(a) $E\left[\operatorname{Var}_{Q_{T}}(L)\right]<+\infty, \quad \forall T \geq 0$, where $\operatorname{Var}_{Q_{T}}(L)$ denotes the total variation of $L$ on $Q_{T}=$ $[0, T] \times[0,1]$.

(b) $L$ is adapted in the sense that for any bounded measurable mapping $\psi$ :

$$
\int_{0}^{t} \int_{0}^{1} \psi(s, x) L(d s, d x) \text { is } \mathcal{F}_{t} \text { measurable. }
$$

(iii) $(u, L)$ solves the parabolic SPDE with reflection in the following sense $(<\cdot, \cdot\rangle$ denotes the

\footnotetext{
${ }^{1}$ School of Mathematics, University of Manchester, Oxford Road, Manchester M13 9PL, England, U.K. Email: tzhang@maths.man.ac.uk
} 
scalar product in $\left.L^{2}([0,1], R)\right): \forall t \in \mathbb{R}_{+}, \phi \in C_{0}^{2}([0,1] \rightarrow R)$ with $\phi(0)=\phi(1)=0$,

$$
\begin{aligned}
& <u(t), \phi>-\int_{0}^{t}<u(s), \phi^{\prime \prime}>d s+\int_{0}^{t}<g(u(s)), \phi^{\prime}>d s \\
= & <u(0), \phi>+\sum_{k=1}^{m} \int_{0}^{t}<\sigma_{k}(u(s)), \phi>d B_{k}(s)+\int_{0}^{t} \int_{0}^{1} \phi(x) L(d s, d x) \text { a.s }
\end{aligned}
$$

where $u(t):=u(t, \cdot)$.

(iv) for any $T>0, \int_{0}^{T} \int_{0}^{1} u(t, x) L(d t, d x)=0$.

Reflected SPDEs have been studied by a number of people. The existence and uniqueness of solutions of SPDEs with reflection driven by additive space-time white noise were obtained by Nualart and Pardoux in [NP] (PTRF 1992). The existence of solutions of reflected SPDEs driven by multiplicative space-time white noise were established by Donati-Martin and Pardoux in [DP1](in PTRF 1993). However, the uniqueness was left open in [DP1]. T.Xu and T.Zhang in [XZ] proved the uniqueness of the solution and also obtained a large deviation principle. Various properties of the solution of reflected SPDEs were studied in [DMZ],[DP2],[HP], [ZA] and [Z]. SPDEs with reflection can also be used to model the evolution of random interfaces near a hard wall. It was proved by $\mathrm{T}$. Funaki and S. Olla in [FO] that the fluctuations of a $\nabla \phi$ interface model near a hard wall converge in law to the stationary solution of a SPDE with reflection. For stochastic Cahn-Hilliard equations with reflection, please see [DZ]. In the above mentioned literature, all the coefficients are assumed to be Lipschitz and of linear growth. However, stochastic Burgers type equations driven by space-time white noise were studied in [GN]. For general references of SPDEs, we refer the reader to $[\mathrm{DZ}]$ and $[\mathrm{PR}]$.

The purpose of this paper is to establish the existence and uniqueness of solutions of stochastic Burgers-type equations with reflection (1.1). As it is well known, the main problem for stochastic Burgers-type equations is to treat the highly non-linear term $\frac{\partial g(u(t, x))}{\partial x}$ which involves the derivative of the solution. In the reflected case, additional difficulties come from the singularities caused by the random measure term $L(d t, d x)$ which plays the role of local time preventing the solution from being negative. We will distinguish two separate situations. The first one is when the initial function $u_{0}$ belongs to the Sobolev space $H^{1}(0,1)$. In this case we will obtain the existence and uniqueness of the solution. The method is to use the approximations of penalized stochastic Burgers-type equations. To prove the convergence of the solutions of the penalized SPDEs, we need to establish a number of estimates for the approximating solutions. Our approach is inspired by the work in $[\mathrm{HP}]$. The uniqueness is proved through a finite dimensional approximations because Ito's formula can not be directly applied to the stochastic Burgers-type equations with reflection. The second case is when the initial function $u_{0}$ just belongs to the usual $L^{2}([0,1])$-space. We will then carry out another layer of approximations of the initial condition. In this case we are only able to obtain the existence of a generalized solution. The uniqueness is still open.

We introduce the following hypotheses on the coefficients.

(A.1) $\sigma=\left(\sigma_{k}(\cdot), k=1, \ldots, m\right): R \rightarrow R^{m}$ satisfies the Lipschitz condition

$$
\sum_{k=1}^{m}\left|\sigma_{k}\left(z_{1}\right)-\sigma_{2}\left(z_{2}\right)\right| \leq C\left|z_{1}-z_{2}\right| .
$$

$\left(\right.$ A.2) $g(\cdot): R \rightarrow R$ is a $C^{1}$-function satisfying

$$
\left|g^{\prime}(z)\right| \leq C(1+|z|)
$$


Remark 1.1. Note that (1.3) implies

$$
\left|g\left(z_{1}\right)-g\left(z_{2}\right)\right| \leq C\left(\left|z_{1}\right|+\left|z_{2}\right|\right)\left|z_{1}-z_{2}\right| .
$$

Here are some nations used throughout the paper. Let $H=L^{2}([0,1], R)$ be the usual $L^{2}$-space with norm $|\cdot|_{H}$ or just $|\cdot|$ and inner product $\langle\cdot, \cdot\rangle$. Denote by $V$ the Sobolev space of order one, i.e., $V$ is the completion of $C_{0}^{\infty}(0,1)$ under the norm $\|u\|^{2}=\int_{0}^{1}\left(\frac{\partial u}{\partial x}\right)^{2} d x$. The corresponding inner product will be denoted by $\langle<\cdot, \cdot\rangle>$. Remark that $V=\left\{u \in H^{1}(0,1): u(0)=u(1)=0\right\}$. $H_{0}^{2}(0,1)$ will denote the Sobolev space of order $2: H_{0}^{2}(0,1)=\left\{u \in V ; \frac{\partial^{2} u}{\partial x^{2}} \in H\right\}$. The norm of $H_{0}^{2}(0,1)$ is denoted by $|\cdot|_{H_{0}^{2}(0,1)}$.

The rest of the paper is organized as follows. In Section 2, we introduce the penalized stochastic Burgers type equations. A number of preliminary estimates will be established. We will also present a comparison theorem which is of independent interest. In Section 3, we prove the main result of existence and uniqueness under the condition that the initial function belongs to the Sobolev space $H_{0}^{1}(0,1)$. In Section 4, we extend the existence result to the general case where the initial function is in the $L^{2}$ space.

\section{Penalized Equations}

For $n \geq 1$, consider the penalized stochastic Burgers-type equation:

$$
\begin{aligned}
u^{n}(t, x)= & u(0, x)+\int_{0}^{t} \frac{\partial^{2} u^{n}(s, x)}{\partial x^{2}} d s+\int_{0}^{t} \sigma\left(u^{n}(s, x)\right) d B(s) \\
& +\int_{0}^{t} \frac{\partial g\left(u^{n}(s, x)\right)}{\partial x} d s+n \int_{0}^{t} u^{n}(s, x)^{-} d s, x \in[0,1], \\
u^{n}(0, x)= & u_{0}(x), \quad x \in[0,1], \\
u^{n}(t, 0)= & u^{n}(t, 1)=0 .
\end{aligned}
$$

It was proved in [GN] that there exists a unique solution $u^{n}(t, x)$ to equation (2.1). In this section, we prepare a number of necessary estimates for the sequence $u^{n}, n \geq 1$. We will also prove a comparison theorem which is of independent interest. We start with the following result.

Lemma 2.1. For $T>0$, the following estimates hold

$$
\begin{gathered}
\sup _{n}\left\{E\left[\sup _{0 \leq t \leq T}\left|u^{n}(t)\right|_{H}^{2}\right]+E\left[\int_{0}^{T}\left\|u^{n}(t)\right\|^{2} d t\right]\right\}<\infty, \\
n E\left[\int_{0}^{T}\left|u^{n}(t)^{-}\right|_{H}^{2} d t\right] \leq K,
\end{gathered}
$$

for some constant $K$, independent of $n$. Furthermore, the following stronger conclusion is valid.

$$
\begin{gathered}
\sup _{n}\left\{E\left[\sup _{0 \leq t \leq T}\left|u^{n}(t)\right|_{H}^{4}\right]+E\left[\left(\int_{0}^{T}\left\|u^{n}(t)\right\|^{2} d t\right)^{2}\right]\right\}<\infty, \\
E\left[\left(n \int_{0}^{T}\left|u^{n}(t)^{-}\right|_{H}^{2} d t\right)^{2}\right] \leq K_{1},
\end{gathered}
$$

where $K_{1}$ is a constant. 
Proof. In the sequel, we will write $u^{n}(t)$ for the function $u^{n}(t, \cdot)$. Applying Ito's formula, we have

$$
\begin{aligned}
\left|u^{n}(t)\right|_{H}^{2} & =\left|u^{n}(0)\right|_{H}^{2}+2 \int_{0}^{t} d s \int_{0}^{1} u^{n}(s, x) \frac{\partial^{2} u^{n}(s, x)}{\partial x^{2}} d x \\
& +2 \sum_{k=1}^{m} \int_{0}^{t}<u^{n}(s), \sigma_{k}\left(u^{n}(s)\right)>d B_{k}(s)+2 \int_{0}^{t} \int_{0}^{1} u^{n}(s, x) \frac{\partial g\left(u^{n}(s, x)\right)}{\partial x} d x d s \\
& -2 n \int_{0}^{t}\left|u^{n}(s)^{-}\right|_{H}^{2} d s+\sum_{k=1}^{m} \int_{0}^{t}\left|\sigma_{k}\left(u^{n}(s)\right)\right|_{H}^{2} d s .
\end{aligned}
$$

Observe that

$$
2 \int_{0}^{t} d s \int_{0}^{1} u^{n}(s, x) \frac{\partial^{2} u^{n}(s, x)}{\partial x^{2}} d x=-2 \int_{0}^{t}\left\|u^{n}(s)\right\|^{2} d s .
$$

Setting $G(z)=\int_{0}^{z} g(y) d y$ we have

$$
\begin{aligned}
& \int_{0}^{t} \int_{0}^{1} u^{n}(s, x) \frac{\partial g\left(u^{n}(s, x)\right)}{\partial x} d x d s=-\int_{0}^{t} d s \int_{0}^{1} \frac{\partial u^{n}(s, x)}{\partial x} g\left(u^{n}(s, x)\right) d x \\
= & -\int_{0}^{t} d s \int_{0}^{1} \frac{\partial G\left(u^{n}(s, x)\right)}{\partial x} d x=-\int_{0}^{t} d s\left[G\left(u^{n}(s, 1)\right)-G\left(u^{n}(s, 0)\right)\right]=0 .
\end{aligned}
$$

Rearranging terms in (2.6) yields

$$
\begin{aligned}
& \sup _{0 \leq r \leq t}\left|u^{n}(r)\right|_{H}^{2}+n \int_{0}^{t}\left|u^{n}(s)^{-}\right|_{H}^{2} d s+\int_{0}^{t} \|\left. u^{n}(s)\right|^{2} d s \\
\leq & C\left|u^{n}(0)\right|_{H}^{2}+C \sum_{k=1}^{m} \sup _{0 \leq r \leq t}\left|\int_{0}^{r}<u^{n}(s), \sigma_{k}\left(u^{n}(s)\right)>d B_{k}(s)\right| \\
+ & C \sum_{k=1}^{m} \int_{0}^{t}\left|\sigma_{k}\left(u^{n}(s)\right)\right|_{H}^{2} d s .
\end{aligned}
$$

By the Burkhölder's inequality,

$$
\begin{aligned}
& C \sum_{k=1}^{m} E\left[\sup _{0 \leq r \leq t}\left|\int_{0}^{r}<u^{n}(s), \sigma_{k}\left(u^{n}(s)\right)>d B_{k}(s)\right|\right] \\
\leq & C_{1} \sum_{k=1}^{m} E\left[\left(\int_{0}^{t}<u^{n}(s), \sigma_{k}\left(u^{n}(s)\right)>^{2} d s\right)^{\frac{1}{2}}\right] \\
\leq & C_{1} \sum_{k=1}^{m} E\left[\left(\sup _{0 \leq r \leq t}\left|u^{n}(r)\right|_{H}\right)\left(\int_{0}^{t}\left|\sigma_{k}\left(u^{n}(s)\right)\right|_{H}^{2} d s\right)^{\frac{1}{2}}\right] \\
\leq & \frac{1}{2} E\left[\left(\sup _{0 \leq r \leq t}\left|u^{n}(r)\right|_{H}^{2}\right)\right]+C_{2} \sum_{k=1}^{m} E\left[\int_{0}^{t}\left|\sigma_{k}\left(u^{n}(s)\right)\right|_{H}^{2} d s\right] .
\end{aligned}
$$

By the linear growth of $\sigma$ and (2.8) we obtain from (2.7) that

$$
\begin{aligned}
& E\left[\sup _{0 \leq r \leq t}\left|u^{n}(r)\right|_{H}^{2}\right]+n E\left[\int_{0}^{t}\left|u^{n}(s)^{-}\right|_{H}^{2} d s\right]+E\left[\int_{0}^{t} \| u^{n}(s)||^{2} d s\right] \\
\leq & C\left|u^{n}(0)\right|_{H}^{2}+C E\left[\int_{0}^{t}\left(1+\left|u^{n}(s)\right|_{H}^{2}\right) d s\right] .
\end{aligned}
$$


Invoking the Gronwall's inequality, this implies (2.2) and (2.3). Squaring the inequality (2.7) and following the similar procedure we can prove (2.4) and (2.5).

Let $b_{1}(\cdot), b_{2}(\cdot)$ be two real-valued measurable functions. Consider the solutions of the following stochastic Burgers-type equations for $i=1,2$,

$$
\begin{aligned}
u_{i}(t, x)= & u_{i}(0, x)+\int_{0}^{t} \frac{\partial^{2} u_{i}(s, x)}{\partial x^{2}} d s+\int_{0}^{t} \sigma\left(u_{i}(s, x)\right) d B(s) \\
& +\int_{0}^{t} \frac{\partial g\left(u_{i}(s, x)\right)}{\partial x} d s+\int_{0}^{t} b_{i}\left(u_{i}(s, x)\right) d s . \\
u_{i}(t, 0)= & u_{i}(t, 1)=0 .
\end{aligned}
$$

Next result is a comparison theorem for the stochastic Burgers-type equations, which is of independent interest.

Theorem 2.1. Assume one of the functions $b_{i}$, say $b_{2}$, is Lipschitz continuous. If $u_{1}(0, x) \leq$ $u_{2}(0, x)$ and $b_{1}\left(u_{1}(t, x)\right) \leq b_{2}\left(u_{1}(t, x)\right)$ for $x \in[0,1]$ a.e., then we have $u_{1}(t, x) \leq u_{2}(t, x), x \in$ $[0,1]$, a.e. for every $t \in[0, T]$.

Proof. For $n \geq 1$, define functions $\psi_{n}(z), f_{n}(y)$ as follows (see [DP1]).

$$
\begin{gathered}
\psi_{n}(z)= \begin{cases}0 & \text { if } z \leq 0, \\
2 n z & \text { if } 0 \leq z \leq \frac{1}{n} \\
2 & \text { if } z>\frac{1}{n}\end{cases} \\
f_{n}(y)= \begin{cases}0 & \text { if } y \leq 0, \\
\int_{0}^{y} d z_{1} \int_{0}^{z_{1}} \psi_{n}(z) d z & \text { if } y>0 .\end{cases}
\end{gathered}
$$

We have

$$
f_{n}^{\prime}(y)= \begin{cases}0 & \text { if } y \leq 0 \\ n y^{2} & \text { if } 0 \leq y \leq \frac{1}{n} \\ 2 y-\frac{1}{n} & \text { if } y>\frac{1}{n}\end{cases}
$$

It is clear that $f_{n}(y) \uparrow\left(y^{+}\right)^{2}$ as $n \rightarrow \infty$. For $h \in H$, set

$$
F_{n}(h)=\int_{0}^{1} f_{n}(h(x)) d x .
$$

$F_{n}$ has the following derivatives for $h_{1}, h_{2} \in H$,

$$
\begin{gathered}
F_{n}^{\prime}(h)\left(h_{1}\right)=\int_{0}^{1} f_{n}^{\prime}(h(x)) h_{1}(x) d x \\
F_{n}^{\prime \prime}(h)\left(h_{1}, h_{2}\right)=\int_{0}^{1} f_{n}^{\prime \prime}(h(x)) h_{1}(x) h_{2}(x) d x .
\end{gathered}
$$

Setting $J(t)=\exp \left\{-\lambda \int_{0}^{t}\left(\left\|u_{1}(r, \cdot)\right\|^{2}+\left\|u_{2}(r, \cdot)\right\|^{2}\right) d r\right\}$ and applying Ito's formula in the framework 
of the Gelfand triple $V \subset H \subset V^{*}$ (see $[\mathrm{P}]$ and $[\mathrm{PR}]$ ) we obtain

$$
\begin{aligned}
& F_{n}\left(u_{1}(t)-u_{2}(t)\right) J(t) \\
= & F_{n}\left(u_{1}(0)-u_{2}(0)\right)-\lambda \int_{0}^{t} J(s)\left(\left\|u_{1}(s, \cdot)\right\|^{2}+\left\|u_{2}(s, \cdot)\right\|^{2}\right) F_{n}\left(u_{1}(s)-u_{2}(s)\right) d s \\
& +\int_{0}^{t} J(s) F_{n}^{\prime}\left(u_{1}(s)-u_{2}(s)\right)\left(\frac{\partial^{2}\left(u_{1}(s)-u_{2}(s)\right)}{\partial x^{2}}\right) d s \\
& +\int_{0}^{t} J(s) F_{n}^{\prime}\left(u_{1}(s)-u_{2}(s)\right)\left(\frac{\partial\left(g\left(u_{1}(s)\right)-g\left(u_{2}(s)\right)\right)}{\partial x}\right) d s \\
& +\int_{0}^{t} J(s) F_{n}^{\prime}\left(u_{1}(s)-u_{2}(s)\right)\left(b_{1}\left(u_{1}(s)\right)-b_{2}\left(u_{2}(s)\right)\right) d s \\
& +\int_{0}^{t} J(s) F_{n}^{\prime}\left(u_{1}(s)-u_{2}(s)\right)\left(\sigma\left(u_{1}(s)\right)-\sigma\left(u_{2}(s)\right)\right) d B_{s} \\
& +\frac{1}{2} \sum_{k=1}^{m} \int_{0}^{t} J(s) F_{n}^{\prime \prime}\left(u_{1}(s)-u_{2}(s)\right)\left(\sigma_{k}\left(u_{1}(s)\right)-\sigma_{k}\left(u_{2}(s)\right), \sigma_{k}\left(u_{1}(s)\right)-\sigma_{k}\left(u_{2}(s)\right)\right) d s \\
=: & I_{n}^{1}+I_{n}^{2}+I_{n}^{3}+I_{n}^{4}+I_{n}^{5}+I_{n}^{6}+I_{n}^{7} .
\end{aligned}
$$

Observe that

$$
\begin{aligned}
I_{n}^{3} & =\int_{0}^{t} J(s) \int_{0}^{1} f_{n}^{\prime}\left(u_{1}(s, x)-u_{2}(s, x)\right) \frac{\partial^{2}\left(u_{1}(s, x)-u_{2}(s, x)\right)}{\partial x^{2}} d x d s \\
& =-\int_{0}^{t} J(s) \int_{0}^{1} f_{n}^{\prime \prime}\left(u_{1}(s, x)-u_{2}(s, x)\right)\left|\frac{\partial}{\partial x}\left(u_{1}(s, x)-u_{2}(s, x)\right)\right|^{2} d x d s .
\end{aligned}
$$

We note that

$$
\begin{aligned}
& I_{n}^{4} \\
= & -\int_{0}^{t} J(s) \int_{0}^{1} f_{n}^{\prime \prime}\left(u_{1}(s, x)-u_{2}(s, x)\right) \frac{\partial}{\partial x}\left(u_{1}(s, x)-u_{2}(s, x)\right)\left(g\left(u_{1}(s, x)\right)-g\left(u_{2}(s, x)\right)\right) \\
\leq & \frac{1}{2} \int_{0}^{t} J(s) \int_{0}^{1} f_{n}^{\prime \prime}\left(u_{1}(s, x)-u_{2}(s, x)\right)\left|\frac{\partial}{\partial x}\left(u_{1}(s, x)-u_{2}(s, x)\right)\right|^{2} d x d s \\
& +C \int_{0}^{t} J(s) \int_{0}^{1} f_{n}^{\prime \prime}\left(u_{1}(s, x)-u_{2}(s, x)\right)\left|g\left(u_{1}(s, x)\right)-g\left(u_{2}(s, x)\right)\right|^{2} d x d s .
\end{aligned}
$$

In view of (1.3), (1.4) and noticing $f_{n}^{\prime \prime}(y) \leq 2 \chi_{\{y \geq 0\}}$, we have

$$
\begin{aligned}
& \int_{0}^{t} J(s) \int_{0}^{1} f_{n}^{\prime \prime}\left(u_{1}(s, x)-u_{2}(s, x)\right)\left|g\left(u_{1}(s, x)\right)-g\left(u_{2}(s, x)\right)\right|^{2} d x d s \\
\leq & \left.C \int_{0}^{t} J(s)\left(\left|u_{1}(s, \cdot)\right|_{L^{\infty}([0,1])}^{2}+\left|u_{2}(s, \cdot)\right|_{L^{\infty}([0,1])}^{2}\right) \int_{0}^{1}\left(\left(u_{1}(s, x)\right)-u_{2}(s, x)\right)^{+}\right)^{2} d x d s \\
\leq & C \int_{0}^{t} J(s)\left(\left\|u_{1}(s, \cdot)\right\|^{2}+\left\|u_{2}(s, \cdot)\right\|^{2}\right)\left|\left(u_{1}(s)-u_{2}(s)\right)^{+}\right|_{H}^{2} d s .
\end{aligned}
$$

Thus from (2.18) we get that

$$
\begin{aligned}
I_{n}^{4} \leq & \frac{1}{2} \int_{0}^{t} J(s) \int_{0}^{1} f_{n}^{\prime \prime}\left(u_{1}(s, x)-u_{2}(s, x)\right)\left|\frac{\partial}{\partial x}\left(u_{1}(s, x)-u_{2}(s, x)\right)\right|^{2} d x d s \\
& \left.+C \int_{0}^{t} J(s)\left(\left\|u_{1}(s, \cdot)\right\|^{2}+\left\|u_{2}(s, \cdot)\right\|^{2}\right) \int_{0}^{1}\left(\left(u_{1}(s, x)\right)-u_{2}(s, x)\right)^{+}\right)^{2} d s .
\end{aligned}
$$


Taking into account the assumptions on $b_{1}, b_{2}, I_{n}^{5}$ can be bounded as

$$
\begin{aligned}
I_{n}^{5}= & \int_{0}^{t} J(s) \int_{0}^{1} f_{n}^{\prime}\left(u_{1}(s, x)-u_{2}(s, x)\right)\left(b_{1}\left(u_{1}(s, x)\right)-b_{2}\left(u_{1}(s, x)\right)\right) d x d s \\
& +\int_{0}^{t} J(s) \int_{0}^{1} f_{n}^{\prime}\left(u_{1}(s, x)-u_{2}(s, x)\right)\left(b_{2}\left(u_{1}(s, x)\right)-b_{2}\left(u_{2}(s, x)\right)\right) d x d s \\
\leq & C \int_{0}^{t} J(s) \int_{0}^{1}\left(\left(u_{1}(s, x)-u_{2}(s, x)\right)^{+}\right)^{2} d x d s .
\end{aligned}
$$

By the Lipschtiz condition of $\sigma_{k}$, it is seen that

$$
I_{n}^{7} \leq C \int_{0}^{t} J(s) \int_{0}^{1}\left(\left(u_{1}(s, x)-u_{2}(s, x)\right)^{+}\right)^{2} d x d s
$$

Putting the above estimates together it follows that

$$
\begin{aligned}
& F_{n}\left(u_{1}(t)-u_{2}(t)\right) J(t) \\
\leq & F_{n}\left(u_{1}(0)-u_{2}(0)\right)-\lambda \int_{0}^{t} J(s)\left(\left\|u_{1}(s, \cdot)\right\|^{2}+\left\|u_{2}(s, \cdot)\right\|^{2}\right) F_{n}\left(u_{1}(s)-u_{2}(s)\right) d s \\
& +C \int_{0}^{t} J(s) \int_{0}^{1}\left(\left(u_{1}(s, x)-u_{2}(s, x)\right)^{+}\right)^{2} d x d s \\
& +\int_{0}^{t} J(s) F_{n}^{\prime}\left(u_{1}(s)-u_{2}(s)\right)\left(\sigma\left(u_{1}(s)\right)-\sigma\left(u_{2}(s)\right)\right) d B_{s} \\
& \left.+C \int_{0}^{t} J(s)\left(\left\|u_{1}(s, \cdot)\right\|^{2}+\left\|u_{2}(s, \cdot)\right\|^{2}\right)\left(\left(u_{1}(s, x)\right)-u_{2}(s, x)\right)^{+}\right)^{2} d s .
\end{aligned}
$$

Take expectation, let $n \rightarrow \infty$ and choose $\lambda>0$ sufficiently large to get

$$
E\left[J(t) \int_{0}^{1}\left(\left(u_{1}(t, x)-u_{2}(t, x)\right)^{+}\right)^{2} d x\right] \leq \int_{0}^{t} d s E\left[J(s) \int_{0}^{1}\left(\left(u_{1}(s, x)-u_{2}(s, x)\right)^{+}\right)^{2} d x\right] .
$$

Gronwall's inequality yields that

$$
E\left[J(t) \int_{0}^{1}\left(\left(u_{1}(t, x)-u_{2}(t, x)\right)^{+}\right)^{2} d x\right]=0,
$$

which completes the proof of the theorem.

We need the following result.

Lemma 2.2. Assume $u_{0} \in V$. For sufficiently large $\lambda$, we have

$$
\begin{gathered}
\sup _{n} E\left[\sup _{0 \leq t \leq T}\left\{\exp \left\{-\lambda \int_{0}^{t}\left\|u^{n}(s)\right\|^{2} d s\right\}\left\|u^{n}(t)\right\|^{2}\right\}\right]<\infty, \\
\sup _{n} E\left[\int_{0}^{T} \exp \left\{-\lambda \int_{0}^{t}\left\|u^{n}(r)\right\|^{2} d r\right\}\left|u^{n}(t)\right|_{H_{0}^{2}(0,1)}^{2} d t\right]<\infty .
\end{gathered}
$$


Proof. By Ito's formula, we have

$$
\begin{aligned}
& \left\|u^{n}(t)\right\|^{2} \exp \left\{-\lambda \int_{0}^{t}\left\|u^{n}(s)\right\|^{2} d s\right\} \\
= & \left\|u_{0}\right\|^{2}-\lambda \int_{0}^{t}\left\|u^{n}(s)\right\|^{4} \exp \left\{-\lambda \int_{0}^{s}\left\|u^{n}(r)\right\|^{2} d r\right\} d s \\
+ & 2 \int_{0}^{t} \exp \left\{-\lambda \int_{0}^{s}\left\|u^{n}(r)\right\|^{2} d r\right\}<<u^{n}(s), \frac{\partial^{2} u^{n}(s)}{\partial x^{2}}>>d s \\
+ & 2 \sum_{k=1}^{m} \int_{0}^{t} \exp \left\{-\lambda \int_{0}^{s}\left\|u^{n}(r)\right\|^{2} d r\right\}<<u^{n}(s), \sigma_{k}\left(u^{n}(s)\right)>>d B_{k}(s) \\
& +2 \int_{0}^{t} \exp \left\{-\lambda \int_{0}^{s}\left\|u^{n}(r)\right\|^{2} d r\right\}<<u^{n}(s), \frac{\partial g\left(u^{n}(s)\right)}{\partial x}>>d s \\
+ & 2 n \int_{0}^{t} \exp \left\{-\lambda \int_{0}^{s}\left\|u^{n}(r)\right\|^{2} d r\right\}<<u^{n}(s), u^{n}(s)^{-}>>d s \\
+ & \sum_{k=1}^{m} \int_{0}^{t} \exp \left\{-\lambda \int_{0}^{s}\left\|u^{n}(r)\right\|^{2} d r\right\}\left\|\sigma_{k}\left(u^{n}(s)\right)\right\|^{2} d s .
\end{aligned}
$$

Observe that

$$
<<u^{n}(s), \frac{\partial^{2} u^{n}(s)}{\partial x^{2}}>>=-\left|u^{n}(s)\right|_{H_{0}^{2}(0,1)}^{2}, \quad\left\|\sigma_{k}\left(u^{n}(s)\right)\right\| \leq C\left(1+\| u^{n}(s)||\right),
$$

and

$$
<<u^{n}(s), u^{n}(s)^{-}>>=\int_{0}^{1} \frac{\partial u^{n}(s, x)}{\partial x} \frac{\partial u^{n}(s, x)^{-}}{\partial x} d x=-\left\|u^{n}(s)^{-}\right\|^{2} .
$$

Moreover,

$$
\begin{aligned}
& <<u^{n}(s), \frac{\partial g\left(u^{n}(s)\right)}{\partial x}>>=\int_{0}^{1} \frac{\partial u^{n}(s, x)}{\partial x} \frac{\partial}{\partial x}\left[\frac{\partial g\left(u^{n}(s, x)\right)}{\partial x}\right] d x \\
= & -\int_{0}^{1} \frac{\partial^{2} u^{n}(s, x)}{\partial x^{2}} g^{\prime}\left(u^{n}(s, x)\right) \frac{\partial u^{n}(s, x)}{\partial x} d x \\
\leq & \frac{1}{4} \int_{0}^{1}\left(\frac{\partial^{2} u^{n}(s, x)}{\partial x^{2}}\right)^{2} d x+C \int_{0}^{1}\left|g^{\prime}\left(u^{n}(s, x)\right)\right|^{2}\left(\frac{\partial u^{n}(s, x)}{\partial x}\right)^{2} d x \\
\leq & \frac{1}{4}\left|u^{n}(s)\right|_{H_{0}^{2}(0,1)}^{2}+C\left(1+\left\|u^{n}(s)\right\|_{\infty}^{2}\right)\left\|u^{n}(s)\right\|^{2} \\
\leq & \frac{1}{4}\left|u^{n}(s)\right|_{H_{0}^{2}(0,1)}^{2}+C\left\|u^{n}(s)\right\|^{4}+C\left\|u^{n}(s)\right\|^{2} .
\end{aligned}
$$

By virtue of (2.29)-(2.31), choosing sufficiently large $\lambda$ and rearranging terms in (2.28) yield

$$
\begin{aligned}
& \left\|u^{n}(t)\right\|^{2} \exp \left\{-\lambda \int_{0}^{t}\left\|u^{n}(s)\right\|^{2} d s\right\} \\
+ & \frac{1}{2} \int_{0}^{t} \exp \left\{-\lambda \int_{0}^{s}\left\|u^{n}(r)\right\|^{2} d r\right\}\left|u^{n}(s)\right|_{H_{0}^{2}(0,1)}^{2} d s \\
+ & 2 n \int_{0}^{t} \exp \left\{-\lambda \int_{0}^{s}\left\|u^{n}(r)\right\|^{2} d r\right\}\left\|u^{n}(s)^{-}\right\|^{2} d s \\
\leq & C\|u(0)\|^{2}+C \int_{0}^{t} \exp \left\{-\lambda \int_{0}^{s}\left\|u^{n}(r)\right\|^{2} d r\right\}\left\|u^{n}(s)\right\|^{2} d s \\
+ & \sum_{k=1}^{m} \int_{0}^{t} \exp \left\{-\lambda \int_{0}^{s}\left\|u^{n}(r)\right\|^{2} d r\right\}<<u^{n}(s), \sigma_{k}\left(u^{n}(s)\right)>>d B_{k}(s) .
\end{aligned}
$$


By the Burkhölder's inequality,

$$
\begin{aligned}
& C \sum_{k=1}^{m} E\left[\sup _{0 \leq t \leq l} \mid \int_{0}^{t} \exp \left\{-\lambda \int_{0}^{s}\left\|u^{n}(r)\right\|^{2} d r\right\}<<u^{n}(s), \sigma_{k}\left(u^{n}(s)\right)>>d B_{k}(s) \|\right] \\
\leq & C_{1} \sum_{k=1}^{m} E\left[\left(\int_{0}^{l} \exp \left\{-2 \lambda \int_{0}^{s}\left\|u^{n}(r)\right\|^{2} d r\right\}<<u^{n}(s), \sigma_{k}\left(u^{n}(s)\right)>>^{2} d s\right)^{\frac{1}{2}}\right] \\
\leq & C_{1} \sum_{k=1}^{m} E\left[\left(\sup _{0 \leq t \leq l} \exp \left\{-\frac{1}{2} \lambda \int_{0}^{t}\left\|u^{n}(s)\right\|^{2} d s\right\}\left\|u^{n}(t)\right\|\right)\left(\int_{0}^{t} \exp \left\{-\lambda \int_{0}^{s}\left\|u^{n}(r)\right\|^{2} d r\right\}\left\|\sigma_{k}\left(u^{n}(s)\right)\right\|^{2} d s\right)^{\frac{1}{2}}\right] \\
\leq & \frac{1}{2} E\left[\left(\sup _{0 \leq t \leq l} \exp \left\{-\lambda \int_{0}^{t}\left\|u^{n}(s)\right\|^{2} d s\right\}\left\|u^{n}(t)\right\|^{2}\right)\right] \\
& +C_{2} \sum_{k=1}^{m} E\left[\int_{0}^{l} \exp \left\{-\lambda \int_{0}^{s}\left\|u^{n}(r)\right\|^{2} d r\right\}\left\|\sigma_{k}\left(u^{n}(s)\right)\right\|^{2} d s\right] .
\end{aligned}
$$

Substituting (2.33) into (2.32) and taking expectation we obtain that

$$
\begin{aligned}
& E\left[\sup _{0 \leq t \leq l}\left\|u^{n}(t)\right\|^{2} \exp \left\{-\lambda \int_{0}^{t}\left\|u^{n}(s)\right\|^{2} d s\right\}\right] \\
+ & \frac{1}{2} E\left[\int_{0}^{l} \exp \left\{-\lambda \int_{0}^{s}\left\|u^{n}(r)\right\|^{2} d r\right\}\left|u^{n}(s)\right|_{H_{0}^{2}(0,1)}^{2} d s\right] \\
+ & 2 n E\left[\int_{0}^{l} \exp \left\{-\lambda \int_{0}^{s}\left\|u^{n}(r)\right\|^{2} d r\right\}\left\|u^{n}(s)^{-}\right\|^{2} d s\right] \\
\leq & C E\left[\left\|u_{0}\right\|^{2}\right]+C E\left[\int_{0}^{l} \exp \left\{-\lambda \int_{0}^{s}\left\|u^{n}(r)\right\|^{2} d r\right\}\left(1+\left\|u^{n}(s)\right\|^{2}\right) d s\right] .
\end{aligned}
$$

This yields (2.26) and (2.27) by invoking Gronwall's inequality.

Set $Q=[0, T] \times[0,1]$. We have

\section{Lemma 2.3.}

$$
\sup _{n} E\left[\left(n\left\|\left(u^{n}\right)^{-}\right\|_{L^{1}(Q)}\right)^{2}\right]<\infty .
$$

Proof. Applying Ito's formula, we have

$$
\begin{aligned}
& \left|u^{n}(t)-1\right|_{H}^{2} \\
= & \left|u^{n}(0)-1\right|_{H}^{2}+2 \int_{0}^{t} d s \int_{0}^{1}\left(u^{n}(s, x)-1\right) \frac{\partial^{2} u^{n}(s, x)}{\partial x^{2}} d x \\
+ & 2 \sum_{k=1}^{m} \int_{0}^{t}<u^{n}(s)-1, \sigma_{k}\left(u^{n}(s)\right)>d B_{k}(s) \\
+ & 2 \int_{0}^{t} \int_{0}^{1}\left(u^{n}(s, x)-1\right) \frac{\partial g\left(u^{n}(s, x)\right)}{\partial x} d x d s \\
+ & 2 n \int_{0}^{t}<u^{n}(s)-1, u^{n}(s)^{-}>d s+\sum_{k=1}^{m} \int_{0}^{t}\left|\sigma_{k}\left(u^{n}(s)\right)\right|_{H}^{2} d s .
\end{aligned}
$$


Setting $G(z)=\int_{0}^{z} g(y) d y$ we have

$$
\begin{aligned}
& \int_{0}^{t} \int_{0}^{1}\left(u^{n}(s, x)-1\right) \frac{\partial g\left(u^{n}(s, x)\right)}{\partial x} d x d s \\
= & -\int_{0}^{t} d s \int_{0}^{1} \frac{\partial G\left(u^{n}(s, x)\right)}{\partial x} d x-\int_{0}^{t} \int_{0}^{1} \frac{\partial g\left(u^{n}(s, x)\right)}{\partial x} d x d s \\
= & -\int_{0}^{t} d s\left[G\left(u^{n}(s, 1)\right)-G\left(u^{n}(s, 0)\right)+g\left(u^{n}(s, 1)\right)-g\left(u^{n}(s, 0)\right)\right]=0 .
\end{aligned}
$$

On the other hand,

$$
-2 n \int_{0}^{t}<u^{n}(s)-1, u^{n}(s)^{-}>d s \geq 2 n \int_{0}^{t} \int_{0}^{1} u^{n}(s, x)^{-} d x d s .
$$

It follows from (2.36), (2.37) and (2.38) that

$$
\begin{aligned}
& E\left[\left(n\left\|\left(u^{n}\right)^{-}\right\|_{L^{1}(Q)}\right)^{2}\right]=E\left[\left(n \int_{0}^{T} \int_{0}^{1} u^{n}(s, x)^{-} d x d s\right)^{2}\right] \\
\leq & C+C E\left[\left|u^{n}(T)-1\right|_{H}^{4}\right]+C E\left[\left|u_{0}-1\right|_{H}^{4}\right]+C E\left[\left(\int_{0}^{T}\left|u^{n}(s)-1\right|_{H}^{2} d s\right)^{2}\right] \\
& +C E\left[\left(\int_{0}^{T}\left\|u^{n}(s)\right\|^{2} d s\right)^{2}\right]+C \sum_{k=1}^{m} E\left[\int_{0}^{T}<u^{n}(s), \sigma_{k}\left(u^{n}(s)\right)>^{2} d s\right] .
\end{aligned}
$$

This implies the desired result (2.35) using Lemma 2.1 .

To show in next section that $u^{n}$ converges in probability in $C([0, T] \rightarrow H)$, we need some more preparations.

Lemma 2.4. For $T>0$, we have

$$
\lim _{n \rightarrow \infty} E\left[\sup _{0 \leq t \leq T}\left|u^{n}(t)^{-}\right|_{H}^{2}\right]=0 .
$$

Proof. For $j \geq 1$, define functions $f_{j}(y)$ as at the beginning of the proof of Theorem 2.1. Then $f_{j}(-y) \uparrow\left(y^{-}\right)^{2}$ as $j \rightarrow \infty$. For $h \in H$, set

$$
R_{j}(h)=\left(\int_{0}^{1} f_{j}(-h(x)) d x\right)^{2} .
$$

$R_{j}$ has the following derivatives, for $h_{1}, h_{2} \in H$,

$$
\begin{aligned}
R_{j}^{\prime}(h)\left(h_{1}\right)= & -2 \int_{0}^{1} f_{j}(-h(x)) d x \int_{0}^{1} f_{j}^{\prime}(-h(x)) h_{1}(x) d x \\
R_{j}^{\prime \prime}(h)\left(h_{1}, h_{2}\right)= & 2 \int_{0}^{1} f_{j}^{\prime}(-h(x)) h_{1}(x) d x \int_{0}^{1} f_{j}^{\prime}(-h(x)) h_{2}(x) d x \\
& +2 \int_{0}^{1} f_{j}(-h(x)) d x \int_{0}^{1} f_{j}^{\prime \prime}(-h(x)) h_{1}(x) h_{2}(x) d x .
\end{aligned}
$$


Applying Ito's formula we obtain

$$
\begin{aligned}
R_{j}\left(u^{n}(t)\right)= & R_{j}\left(u_{0}\right)+\int_{0}^{t} R_{j}^{\prime}\left(u^{n}(s)\right)\left(\frac{\partial^{2} u^{n}(s)}{\partial x^{2}}\right) d s \\
& +\int_{0}^{t} R_{j}^{\prime}\left(u^{n}(s)\right)\left(\frac{\partial g\left(u^{n}(s)\right)}{\partial x}\right) d s \\
& +n \int_{0}^{t} R_{j}^{\prime}\left(u^{n}(s)\right)\left(u^{n}(s)^{-}\right) d s \\
& +\int_{0}^{t} R_{j}^{\prime}\left(u^{n}(s)\right)\left(\sigma\left(u^{n}(s)\right)\right) d B_{s}+\frac{1}{2} \sum_{k=1}^{m} \int_{0}^{t} R_{j}^{\prime \prime}\left(u^{n}(s)\right)\left(\sigma_{k}\left(u^{n}(s)\right), \sigma_{k}\left(u^{n}(s)\right)\right) d s \\
=: & I_{n}^{1}(t)+I_{n}^{2}(t)+I_{n}^{3}(t)+I_{n}^{4}(t)+I_{n}^{5}(t)+I_{n}^{6}(t) .
\end{aligned}
$$

Note that

$$
\begin{aligned}
I_{n}^{2}(t) & =-2 \int_{0}^{t} d s \int_{0}^{1} f_{j}\left(-u^{n}(s, x)\right) d x \int_{0}^{1} f_{j}^{\prime}\left(-u^{n}(s, x)\right) \frac{\partial^{2} u^{n}(s, x)}{\partial x^{2}} d x \\
& =-2 \int_{0}^{t} d s \int_{0}^{1} f_{j}\left(-u^{n}(s, x)\right) d x \int_{0}^{1} f_{j}^{\prime \prime}\left(-u^{n}(s, x)\right)\left|\frac{\partial}{\partial x} u^{n}(s, x)\right|^{2} d x .
\end{aligned}
$$

Setting $U_{j}(z)=\int_{0}^{z} f_{j}^{\prime}(-y) g^{\prime}(y) d y$, we have

$$
\begin{aligned}
I_{n}^{3}(t) & =-2 \int_{0}^{t} d s\left(\int_{0}^{1} f_{j}\left(-u^{n}(s, x)\right) d x\right) \int_{0}^{1} f_{j}^{\prime}\left(-u^{n}(s, x)\right) \frac{\partial g\left(u^{n}(s, x)\right)}{\partial x} d x \\
& =-2 \int_{0}^{t} d s\left(\int_{0}^{1} f_{j}\left(-u^{n}(s, x)\right) d x\right) \int_{0}^{1} \frac{\partial U_{j}\left(u^{n}(s, x)\right)}{\partial x} d x \\
& =-2 \int_{0}^{t} d s\left(\int_{0}^{1} f_{j}\left(-u^{n}(s, x)\right) d x\right)\left(U_{j}\left(u^{n}(s, 1)\right)-U_{j}\left(u^{n}(s, 0)\right)\right)=0 .
\end{aligned}
$$

Since $f_{j}$ and $f_{j}^{\prime}$ are non-negative, we have

$$
I_{n}^{4}(t)=-2 n \int_{0}^{t} d s \int_{0}^{1} f_{j}\left(-u^{n}(s, x)\right) d x \int_{0}^{1} f_{j}^{\prime}\left(-u^{n}(s, x)\right) u^{n}(s, x)^{-} d x \leq 0 .
$$


Using the fact $0 \leq f_{j}^{\prime}(-y) \leq 2 y^{-}$and the Burkholder's inequality we have

$$
\begin{aligned}
& E\left[\sup _{0 \leq t \leq l}\left|I_{n}^{5}(t)\right|\right] \\
\leq & C E\left[\left(\sum_{k=1}^{m} \int_{0}^{l}\left(R_{j}^{\prime}\left(u^{n}(s)\right)\right)\left(\sigma_{k}\left(u^{n}(s)\right)\right)^{2} d s\right)^{\frac{1}{2}}\right] \\
\leq & C E\left[\left(\sum_{k=1}^{m} \int_{0}^{l}\left(\int_{0}^{1} f_{j}\left(-u^{n}(s, x)\right) d x \int_{0}^{1} f_{j}^{\prime}\left(-u^{n}(s, x)\right) \sigma_{k}\left(u^{n}(s, x)\right) d x\right)^{2} d s\right)^{\frac{1}{2}}\right] \\
\leq & C E\left[\sup _{0 \leq t \leq l}\left(\int_{0}^{1} f_{j}\left(-u^{n}(s, x)\right) d x\right)\left(\sum_{k=1}^{m} \int_{0}^{l}\left(\int_{0}^{1} f_{j}^{\prime}\left(-u^{n}(s, x)\right) \sigma_{k}\left(u^{n}(s, x)\right) d x\right)^{2} d s\right)^{\frac{1}{2}}\right] \\
\leq & \frac{1}{2} E\left[\sup _{0 \leq t \leq l}\left(\int_{0}^{1} f_{j}\left(-u^{n}(s, x)\right) d x\right)^{2}\right] \\
& +C E\left[\sum_{k=1}^{m} \int_{0}^{l}\left(\int_{0}^{1} f_{j}^{\prime}\left(-u^{n}(s, x)\right) \sigma_{k}\left(u^{n}(s, x)\right) d x\right)^{2} d s\right] \\
\leq & \frac{1}{2} E\left[\sup _{0 \leq t \leq l} R_{j}(t)\right]+C E\left[\int_{0}^{l}\left(\int_{0}^{1} f_{j}^{\prime}\left(-u^{n}(s, x)\right)\left(1+\left|u^{n}(s, x)\right|\right) d x\right)^{2} d s\right] \\
\leq & \frac{1}{2} E\left[\sup _{0 \leq t \leq l} R_{j}(t)\right]+C E\left[\int_{0}^{l}\left|u^{n}(s)^{-}\right|_{H}^{2} d s\right]+C E\left[\int_{0}^{l}\left|u^{n}(s)^{-}\right|_{H}^{4} d s\right] .
\end{aligned}
$$

By the linear growth of $\sigma_{k}$, we have

$$
\begin{aligned}
I_{n}^{6} \leq & C \int_{0}^{t} d s \sum_{k=1}^{m}\left(\int_{0}^{1} f_{j}^{\prime}\left(-u^{n}(s, x)\right) \sigma_{k}\left(u^{n}(s, x)\right) d x\right)^{2} \\
& +C \int_{0}^{t} d s\left(\int_{0}^{1} f_{j}\left(-u^{n}(s, x)\right) d x\right) \int_{0}^{1} f_{j}^{\prime \prime}\left(-u^{n}(s, x)\right) \sigma_{k}\left(u^{n}(s, x)\right)^{2} d x \\
\leq & C \int_{0}^{t} d s\left(\int_{0}^{1} f_{j}^{\prime}\left(-u^{n}(s, x)\right)\left(1+\left|u^{n}(s, x)\right|\right) d x\right)^{2} \\
& +C \int_{0}^{t} d s\left(\int_{0}^{1} f_{j}\left(-u^{n}(s, x)\right) d x\right) \int_{0}^{1}\left(1+\left|u^{n}(s, x)^{-}\right|\right)^{2} d x \\
\leq & C \int_{0}^{t}\left(\left|u^{n}(s)^{-}\right|_{H}^{2}+\left|u^{n}(s)^{-}\right|_{H}^{4}\right) d s .
\end{aligned}
$$

Putting the above estimates together it follows from (2.43) that

$$
\begin{aligned}
& E\left[\sup _{0 \leq t \leq l} R_{j}\left(u^{n}(t)\right)\right] \\
\leq & C E\left[\int_{0}^{l}\left(\left|u^{n}(s)^{-}\right|_{H}^{2} d s\right]+C E\left[\int_{0}^{l}\left|u^{n}(s)^{-}\right|_{H}^{4} d s\right] .\right.
\end{aligned}
$$

Let $j \rightarrow \infty$ and apply Fatou's lemma to get

$$
E\left[\sup _{0 \leq t \leq T}\left|u^{n}(t)^{-}\right|_{H}^{4}\right] \leq C E\left[\int_{0}^{T}\left(\left|u^{n}(s)^{-}\right|_{H}^{2} d s\right]+C E\left[\int_{0}^{l}\left|u^{n}(s)^{-}\right|_{H}^{4} d s\right] .\right.
$$

Applying Gronwall's inequality yields

$$
E\left[\sup _{0 \leq t \leq T}\left|u^{n}(t)^{-}\right|_{H}^{4}\right] \leq C E\left[\int_{0}^{T}\left(\left|u^{n}(s)^{-}\right|_{H}^{2} d s\right] .\right.
$$


Now the desired conclusion follows from (2.51) and Lemma 2.1.

Set

$$
e_{n}(t)=\exp \left\{-\lambda \int_{0}^{t}\left\|u^{n}(s)\right\|^{2} d s\right\}
$$

We have the following result.

\section{Corollary 2.1.}

$$
\lim _{n \rightarrow \infty} E\left[\sup _{0 \leq t \leq T} e_{n}(t)\left|u^{n}(t)^{-}\right|_{L^{\infty}([0,1])}^{2}\right]=0 .
$$

Proof. By Sobolev imbedding, for every $\epsilon>0$, there exists $C_{\varepsilon}>0$ such that

$$
\begin{aligned}
& E\left[\sup _{0 \leq t \leq T} e_{n}(t)\left|u^{n}(t)^{-}\right|_{L^{\infty}([0,1])}^{2}\right] \\
\leq & \varepsilon E\left[\sup _{0 \leq t \leq T} e_{n}(t) \|\left. u^{n}(t)^{-}\right|^{2}\right]+C_{\varepsilon} E\left[\sup _{0 \leq t \leq T}\left|u^{n}(t)^{-}\right|_{H}^{2}\right] .
\end{aligned}
$$

Letting $n \rightarrow \infty$, it follows from Lemma 2.4 that

$$
\lim _{n \rightarrow \infty} E\left[\sup _{0 \leq t \leq T} e_{n}(t)\left|u^{n}(t)^{-}\right|_{L^{\infty}([0,1])}^{2}\right] \leq C \varepsilon
$$

Send $\varepsilon$ to 0 to prove the Corollary.

\section{Existence and Uniqueness}

Let $u^{n}$ be the solution of the penalized equation (2.1). We will show that the limit of $\left\{u^{n}, n \geq 1\right\}$ is a solution of the stochastic Burgers equation with reflection. We begin with the following lemma.

Lemma 3.1. There exists a constant $\lambda>0$ such that

$$
\begin{aligned}
& \lim _{n, m \rightarrow \infty}\left\{E\left[\sup _{0 \leq t \leq T} \exp \left\{-\lambda \int_{0}^{t}\left(\left\|u^{n}(s)\right\|^{2}+\left\|u^{m}(s)\right\|^{2}\right) d s\right\}\left|u^{n}(t)-u^{m}(t)\right|_{H}^{2}\right]\right. \\
& \left.+E\left[\int_{0}^{T} \exp \left\{-\lambda \int_{0}^{t}\left(\left\|u^{n}(s)\right\|^{2}+\left\|u^{m}(s)\right\|^{2}\right) d s\right\}\left\|u^{n}(t)-u^{m}(t)\right\|^{2} d t\right]\right\}=0
\end{aligned}
$$

Proof. Set

$$
e_{n, m}(t)=\exp \left\{-\lambda \int_{0}^{t}\left\|u^{n}(s)\right\|^{2} d s-\lambda \int_{0}^{t}\left\|u^{m}(s)\right\|^{2} d s\right\} .
$$

Applying Ito's formula, we have for $m \geq n$,

$$
\begin{aligned}
& \left|u^{n}(t)-u^{m}(t)\right|_{H}^{2} e_{n, m}(t) \\
= & -\lambda \int_{0}^{t} e_{n, m}(s)\left(\left\|u^{n}(s)\right\|^{2}+\| u^{m}(s)||^{2}\right)\left|u^{n}(s)-u^{m}(s)\right|_{H}^{2} d s \\
- & 2 \int_{0}^{t} e_{n, m}(s)\left\|u^{n}(s)-u^{m}(s)\right\|^{2} d s \\
+ & 2 \sum_{k=1}^{m} \int_{0}^{t} e_{n, m}(s)<u^{n}(s)-u^{m}(s), \sigma_{k}\left(u^{n}(s)\right)-\sigma_{k}\left(u^{m}(s)\right)>d B_{k}(s) \\
+ & 2 \int_{0}^{t} e_{n, m}(s)<u^{n}(s)-u^{m}(s), \frac{\partial g\left(u^{n}(s)\right)}{\partial x}-\frac{\partial g\left(u^{m}(s)\right)}{\partial x}>d s \\
+ & 2 \int_{0}^{t} e_{n, m}(s)<u^{n}(s)-u^{m}(s), n u^{n}(s)^{-}-m u^{m}(s)^{-}>d s \\
+ & \sum_{k=1}^{m} \int_{0}^{t} e_{n, m}(s)\left|\sigma_{k}\left(u^{n}(s)\right)-\sigma_{k}\left(u^{m}(s)\right)\right|_{H}^{2} d s .
\end{aligned}
$$


For $u, v \in V$, we have

$$
\begin{aligned}
& <u-v, \frac{\partial g(u)}{\partial x}-\frac{\partial g(v)}{\partial x}> \\
= & -\int_{0}^{1} \frac{\partial}{\partial x}(u(x)-v(x))(g(u(x))-g(v(x))) d x \\
\leq & \frac{1}{8} \int_{0}^{1}\left|\frac{\partial}{\partial x}(u(x)-v(x))\right|^{2} d x+C \int_{0}^{1}|g(u(x))-g(v(x))|^{2} d x \\
\leq & \frac{1}{8} \int_{0}^{1}\left|\|u-v\|^{2}+C \int_{0}^{1}\left(\left.u(x)\right|^{2}+|v(x)|^{2}\right)\right| u(x)-\left.v(x)\right|^{2} d x \\
\leq & \frac{1}{8} \int_{0}^{1}\|u-v\|^{2}+C\left(\|u\|^{2}+\|v\|^{2}\right)|u-v|_{H}^{2},
\end{aligned}
$$

where we have used the fact $|u|_{L^{\infty}([0,1])} \leq\|u\|$. Observe that

$$
\begin{aligned}
& 2 \int_{0}^{t} e_{n, m}(s)<u^{n}(s)-u^{m}(s), n u^{n}(s)^{-}-m u^{m}(s)^{-}>d s \\
= & 2 \int_{0}^{t} e_{n, m}(s)<u^{n}(s)-u^{m}(s), n u^{n}(s)^{-}>d s+2 \int_{0}^{t} e_{n, m}(s)<u^{m}(s)-u^{n}(s), m u^{m}(s)^{-}>d s \\
\leq & 2 \int_{0}^{t} e_{n, m}(s)<-u^{m}(s), n u^{n}(s)^{-}>d s+2 \int_{0}^{t} e_{n, m}(s)<-u^{n}(s), m u^{m}(s)^{-}>d s \\
\leq & 2 \int_{0}^{t} e_{n, m}(s) d s \int_{0}^{1} u^{m}(s, x)^{-} n u^{n}(s, x)^{-} d x+2 \int_{0}^{t} e_{n, m}(s) d s \int_{0}^{1} u^{n}(s, x)^{-} m u^{m}(s, x)^{-} d x \\
\leq & 2 \sup _{0 \leq s \leq T} e_{n, m}(s)\left|u^{m}(s, \cdot)^{-}\right|_{L^{\infty}([0,1])}\left(n \|\left.\left(u^{n}\right)^{-}\right|_{L^{1}(Q)}\right) \\
& +2 \sup _{0 \leq s \leq T} e_{n, m}(s)\left|u^{n}(s, \cdot)^{-}\right|_{L^{\infty}([0,1])}\left(m\left\|\left(u^{m}\right)^{-}\right\|_{L^{1}(Q)}\right) .
\end{aligned}
$$

On the other hand, by the Burkholder's inequality we have

$$
\begin{aligned}
& 2 E\left[\sup _{0 \leq t \leq l}\left\{\sum_{k=1}^{m}\left|\int_{0}^{t} e_{n, m}(s)<u^{n}(s)-u^{m}(s), \sigma_{k}\left(u^{n}(s)\right)-\sigma_{k}\left(u^{m}(s)\right)>d B_{k}(s)\right|\right\}\right] \\
\leq & C E\left[\left(\sum_{k=1}^{m} \int_{0}^{l} e_{n, m}(s)^{2}<u^{n}(s)-u^{m}(s), \sigma_{k}\left(u^{n}(s)\right)-\sigma_{k}\left(u^{m}(s)\right)>^{2} d s\right)^{\frac{1}{2}}\right] \\
\leq & C E\left[\left(\sup _{0 \leq t \leq l} e_{n, m}(s)^{\frac{1}{2}}\left|u^{n}(s)-u^{m}(s)\right|_{H}\right)\left(\sum_{k=1}^{m} \int_{0}^{l} e_{n, m}(s)\left|\sigma_{k}\left(u^{n}(s)\right)-\sigma_{k}\left(u^{m}(s)\right)\right|_{H}^{2} d s\right)^{\frac{1}{2}}\right] \\
\leq & \frac{1}{2} E\left[\sup _{0 \leq t \leq l} e_{n, m}(s)\left|u^{n}(s)-u^{m}(s)\right|_{H}^{2}\right]+C E\left[\int_{0}^{l} e_{n, m}(s)\left|u^{n}(s)-u^{m}(s)\right|_{H}^{2} d s\right] .
\end{aligned}
$$


Substitute (3.3), (3.4), (3.5) back into (3.2) to obtain that for large enough $\lambda>0$,

$$
\begin{aligned}
& \left\{E\left[\sup _{0 \leq t \leq l} e_{n, m}(t)\left|u^{n}(t)-u^{m}(t)\right|_{H}^{2}\right]\right. \\
& \left.+E\left[\int_{0}^{l} e_{n, m}(t)\left\|u^{n}(t)-u^{m}(t)\right\|^{2} d t\right]\right\} \\
\leq & C E\left[\int_{0}^{l} e_{n, m}(t)\left|u^{n}(t)-u^{m}(t)\right|_{H}^{2} d t\right] \\
& +C E\left[\sup _{0 \leq s \leq T} e_{n, m}(s)\left|u^{m}(s, \cdot)^{-}\right|_{L^{\infty}([0,1])}\left(n\left\|\left(u^{n}\right)^{-}\right\|_{L^{1}(Q)}\right)\right] \\
& +C E\left[\sup _{0 \leq s \leq T} e_{n, m}(s)\left|u^{n}(s, \cdot)^{-}\right|_{L^{\infty}([0,1])}\left(m\left\|\left(u^{m}\right)^{-}\right\|_{L^{1}(Q)}\right)\right] .
\end{aligned}
$$

Applying Gronwall's inequality and the Hölder's inequality yields that

$$
\begin{aligned}
& \left\{E\left[\sup _{0 \leq t \leq l} e_{n, m}(t)\left|u^{n}(t)-u^{m}(t)\right|_{H}^{2}\right]\right. \\
& \left.+E\left[\int_{0}^{l} e_{n, m}(t)\left\|u^{n}(t)-u^{m}(t)\right\|^{2} d t\right]\right\} \\
\leq & C\left(E\left[\sup _{0 \leq s \leq T} e_{m}(s)\left|u^{m}(s, \cdot)^{-}\right|_{L^{\infty}([0,1])}^{2}\right]\right)^{\frac{1}{2}}\left(E\left[\left(n\left\|\left(u^{n}\right)^{-}\right\|_{L^{1}(Q)}\right)^{2}\right]\right)^{\frac{1}{2}} \\
& +C\left(E\left[\sup _{0 \leq s \leq T} e_{n}(s)\left|u^{n}(s, \cdot)^{-}\right|_{L^{\infty}([0,1])}^{2}\right]\right)^{\frac{1}{2}}\left(E\left[\left(m\left\|\left(u^{m}\right)^{-}\right\|_{L^{1}(Q)}\right)^{2}\right]\right)^{\frac{1}{2}} .
\end{aligned}
$$

This together with Lemma 2.3 and Corollary 2.1 give the results desired.

Lemma 3.2. For any $\delta>0$, we have

$$
\lim _{n, m \rightarrow \infty} P\left(\sup _{0 \leq t \leq T}\left|u^{n}(t)-u^{m}(t)\right|_{H} \geq \delta\right)=0
$$

and

$$
\lim _{n, m \rightarrow \infty} P\left(\int_{0}^{T}\left\|u^{n}(t)-u^{m}(t)\right\|^{2} d t \geq \delta\right)=0 .
$$

Proof. We only prove (3.8). (3.9) can be proved using similar arguments. For any $M>0$, we have

$$
\begin{aligned}
& P\left(\sup _{0 \leq t \leq T}\left|u^{n}(t)-u^{m}(t)\right|_{H} \geq \delta\right) \\
= & P\left(\sup _{0 \leq t \leq T}\left|u^{n}(t)-u^{m}(t)\right|_{H} \geq \delta, \int_{0}^{T}\left(\left\|u^{n}(s)\right\|^{2}+\left\|u^{m}(s)\right\|^{2}\right) d s>M\right) \\
+ & P\left(\sup _{0 \leq t \leq T}\left|u^{n}(t)-u^{m}(t)\right|_{H} \geq \delta, \int_{0}^{T}\left(\left\|u^{n}(s)\right\|^{2}+\left\|u^{m}(s)\right\|^{2}\right) d s \leq M\right) \\
\leq & P\left(\int_{0}^{T}\left(\left\|u^{n}(s)\right\|^{2}+\left\|u^{m}(s)\right\|^{2}\right) d s>M\right) \\
& +P\left(\sup _{0 \leq t \leq T} \exp \left\{-\lambda \int_{0}^{t}\left\|u^{n}(s)\right\|^{2} d s-\left.\lambda \int_{0}^{t}\left\|u^{m}(s)\right\|\right|^{2} d s\right\}\left|u^{n}(t)-u^{m}(t)\right|_{H}^{2} \geq e^{-\lambda M} \delta^{2}\right) \\
\leq & \frac{1}{M} E\left[\int_{0}^{T}\left(\left\|u^{n}(s)\right\|^{2}+\left\|u^{m}(s)\right\|^{2}\right) d s\right] \\
& +e^{\lambda M} \frac{1}{\delta^{2}} E\left[\sup _{0 \leq t \leq T} \exp \left\{-\lambda \int_{0}^{t}\left\|u^{n}(s)\right\|^{2} d s-\lambda \int_{0}^{t}\left\|u^{m}(s)\right\|^{2} d s\right\}\left|u^{n}(t)-u^{m}(t)\right|_{H}^{2}\right]
\end{aligned}
$$


Given $\varepsilon>0$, by Lemma 2.1 we can find $M>0$ so that

$$
\frac{1}{M} E\left[\int_{0}^{T}\left(\left\|u^{n}(s)\right\|^{2}+\left\|u^{m}(s)\right\|^{2}\right) d s\right] \leq \frac{\varepsilon}{2}
$$

for all $n, m \geq 1$. Then by Lemma 3.1 there exists $N_{0}$ such that for $n \geq N_{0}, m \geq N_{0}$,

$$
e^{\lambda M} \frac{1}{\delta^{2}} E\left[\sup _{0 \leq t \leq T} \exp \left\{-\lambda \int_{0}^{t}\left\|u^{n}(s)\right\|^{2} d s-\lambda \int_{0}^{t}\left\|u^{m}(s)\right\|^{2} d s\right\}\left|u^{n}(t)-u^{m}(t)\right|_{H}^{2}\right] \leq \frac{\varepsilon}{2} .
$$

Hence

$$
P\left(\sup _{0 \leq t \leq T}\left|u^{n}(t)-u^{m}(t)\right|_{H} \geq \delta\right) \leq \varepsilon
$$

for all $n \geq N_{0}, m \geq N_{0}$. As $\varepsilon$ is arbitrary, the proof is complete.

Now we are in a position to state the first main result.

Theorem 3.1. Suppose (A.1) and (A.2) hold and $u_{0} \in V$. The reflected SPDE (1.1) admits a unique solution $(u, L)$ that satisfies

$$
E\left[\sup _{0 \leq t \leq T}|u(t)|_{H}^{2}\right]+E\left[\int_{0}^{T}\|u(t)\|_{V}^{2} d t\right]<\infty
$$

for $T>0$. Moreover, the following energy equation holds:

$$
\begin{aligned}
|u(T)|_{H}^{2}+2 \int_{0}^{T}\|u(t)\|_{V}^{2} d t & =|u(0)|_{H}^{2}+2 \sum_{k=1}^{m} \int_{0}^{t} d s<u(s), \sigma_{k}(u(s))>d B_{k}(s) \\
& +\sum_{k=1}^{m} \int_{0}^{t} d s\left|\sigma_{k}(u(s))\right|_{H}^{2} d B_{k}(s) .
\end{aligned}
$$

Remark 3.1. Actually under the assumption of Theorem 3.1, it also holds that

$$
E\left[\sup _{0 \leq t \leq T}\|u(t)\|_{V}^{2}\right]+E\left[\int_{0}^{T}|u(t)|_{H_{0}^{2}(0,1)}^{2} d t\right]<\infty .
$$

Proof. Existence. Let $u^{n}$ be the solution to the penalized equation (2.1). According to Lemma 3.2 , there exists an adapted process $u$ such that for any $T>0, u^{n}$ converges to $u$ in probability in the space $C([0, T], H) \cap L^{2}([0, T], V)$. We will show that $u(t), t \geq 0$ is a solution to equation (1.1). Consider the random measure

$$
L^{(n)}(d t, d x)=n u^{n}(t, x)^{-} d t d x .
$$

For any $T>0$, by Lemma 2.3 we have

$$
\sup _{n} E\left[\left(\operatorname{Var}\left(L^{(n)}\right)([0, T] \times[0,1])\right)^{2}\right] \leq \sup _{n} E\left[\left(n \int_{0}^{T} \int_{0}^{1} u^{n}(t, x)^{-} d t d x\right)^{2}\right]<\infty,
$$

where $\operatorname{Var}\left(L^{(n)}\right)$ denotes the total variation of $L^{(n)}$ on $Q_{T}=[0, T] \times[0,1]$. Let $\mathcal{M}\left(Q_{T}\right)$ denote the Banach space of totally finite signed measures on $Q_{T}$, equipped with the norm of total variation. (3.14) shows that $\left\{L^{(n)}(d t, d x), n \geq 1\right\}$ is bounded in $L^{2}\left(\Omega, \mathcal{M}\left(Q_{T}\right)\right)$, hence relatively compact with respect to the weak* topology in $L^{2}\left(\Omega, \mathcal{M}\left(Q_{T}\right)\right.$ ). Thus, we may assume ( take a subsequence if 
necessary) that $L^{(n)}$ converges to some $L \in L^{2}\left(\Omega, \mathcal{M}\left(Q_{T}\right)\right)$ with respect to the weak* topology. Moreover, it follows from (3.14) that $E\left[(\operatorname{Var}(L)([0, T] \times[0,1]))^{2}\right]<\infty$ for every $T>0$.

Now, take any $\phi \in C_{0}^{2}((0, \infty) \times[0,1] \rightarrow R)$ and use the chain rule and (2.1) to get

$$
\begin{aligned}
& \int_{0}^{t} \int_{0}^{1} \phi(s, x) L^{(n)}(d s, d x) \\
= & <u^{n}(t), \phi(t)>-<u(0), \phi(0)>-\int_{0}^{t}<\frac{\partial \phi(s)}{\partial s}, u^{n}(s)>d s \\
& -\int_{0}^{t}<\frac{\partial^{2} \phi(s)}{\partial x^{2}}, u^{n}(s)>d s \\
& -\sum_{k=1}^{m} \int_{0}^{t}<\phi(s), \sigma_{k}\left(u^{n}(s)\right)>d B_{k}(s)+\int_{0}^{t}<\frac{\partial \phi(s)}{\partial x}, g\left(u^{n}(s)\right)>d s .
\end{aligned}
$$

Choosing a subsequence if necessary, we can assume that $u^{n}$ converges to $u$ almost surely in the space $C([0, T], H) \cap L^{2}([0, T], V)$. Due to the imbedding $V \subset C([0,1])$, for a fixed random parameter $\omega$ we have that $u^{n} \rightarrow u$ in the space $L^{2}([0, T], C([0,1]))$. Thus, $<\frac{\partial \phi(s)}{\partial x}, g\left(u^{n}(s)\right)>\rightarrow<\frac{\partial \phi(s)}{\partial x}, g(u(s))>$ $d s$ - almost everywhere. On the other hand,

$$
\begin{aligned}
& \sup _{n} \int_{0}^{t}<\frac{\partial \phi(s)}{\partial x}, g\left(u^{n}(s)\right)>^{2} d s \\
= & \sup _{n} \int_{0}^{t}\left(\int_{0}^{1} \frac{\partial \phi(s, x)}{\partial x} g\left(u^{n}(s, x)\right) d x\right)^{2} d s \\
\leq & C \sup _{n} \int_{0}^{t}\left(\int_{0}^{1} g\left(u^{n}(s, x)\right) d x\right)^{2} d s \leq C \sup _{n} \int_{0}^{t}\left(\int_{0}^{1}\left(1+\left|u^{n}(s, x)\right|^{2}\right) d x\right)^{2} d s \\
\leq & C\left(1+\sup _{n} \sup _{0 \leq s \leq t}\left|u^{n}(s)\right|_{H}^{4}\right)<\infty .
\end{aligned}
$$

This shows that the family $\left\{<\frac{\partial \phi(s)}{\partial x}, g\left(u^{n}(s)\right)>, s \in[0, t]\right\}_{n \geq 1}$ is uniformly integrable with respect to the Lebesgue measure on the interval $[0, t]$. Putting the above arguments together we deduce that

$$
\lim _{n \rightarrow \infty} \int_{0}^{t}<\frac{\partial \phi(s)}{\partial x}, g\left(u^{n}(s)\right)>d s=\int_{0}^{t}<\frac{\partial \phi(s)}{\partial x}, g(u(s))>d s .
$$

Since every term on the right side of the equation (3.15) converges as $n \rightarrow \infty$, let $n \rightarrow \infty$ to get

$$
\begin{aligned}
& \int_{0}^{t} \int_{0}^{1} \phi(s, x) L(d s, d x) \\
= & <u(t), \phi(t)>-<u(0), \phi(0)>-\int_{0}^{t}<\frac{\partial \phi(s)}{\partial s}, u(s)>d s \\
& -\int_{0}^{t}<\frac{\partial^{2} \phi(s)}{\partial x^{2}}, u(s)>d s \\
& -\sum_{k=1}^{m} \int_{0}^{t}<\phi(s), \sigma_{k}(u(s))>d B_{k}(s)+\int_{0}^{t}<\frac{\partial \phi(s)}{\partial x}, g(u(s))>d s .
\end{aligned}
$$

On the other hand, it follows from Lemma 2.4 and Fatou Lemma we have

$$
\left.\left.E\left[\sup _{0 \leq s \leq T} \mid u(s)^{-}\right)\right|_{H} ^{2}\right] \leq \lim _{n \rightarrow \infty} E\left[\sup _{0 \leq s \leq T}\left|u^{n}(s)^{-}\right|_{H}^{2}\right]=0 .
$$

This shows that for $t \geq 0, u(t, x) \geq 0$ for almost all $x \in[0,1]$. Since $u(t, \cdot) \in V \subset C([0,1]$ by the Sobolev embedding, we have $u(t, x) \geq 0$ for all $x \in[0,1]$. As in the proof of Corollary 2.1, 
by Sobolev imbedding we see that for large enough $\lambda>0, \exp \left(-\lambda \int_{0}^{t}\left\|u^{n}(s)\right\|^{2} d s\right) u^{n}$ converges to $\exp \left(-\lambda \int_{0}^{t}\|u(s)\|^{2} d s\right) u$ in $L^{2}\left(\Omega, C\left(Q_{T}\right)\right) . \mathrm{As}_{\sup _{n}} E\left[\left(\operatorname{Var}\left(L^{(n)}\right)([0, T] \times[0,1])\right)^{2}\right]<\infty$, we deduce that

$$
\begin{aligned}
& E\left[\int_{0}^{T} \int_{0}^{1} \exp \left(-\lambda \int_{0}^{t}\|u(s)\|^{2} d s\right) u(t, x) L(d t, d x)\right] \\
= & \lim _{n \rightarrow \infty} E\left[\int_{0}^{T} \int_{0}^{1} \exp \left(-\lambda \int_{0}^{t}\|u(s)\|^{2} d s\right) u(t, x) L^{(n)}(d t, d x)\right] \\
= & \lim _{n \rightarrow \infty} E\left[\int_{0}^{T} \int_{0}^{1} \exp \left(-\lambda \int_{0}^{t}\left\|u^{n}(s)\right\|^{2} d s\right) u^{n}(t, x) L^{(n)}(d t, d x)\right] \leq 0 .
\end{aligned}
$$

Thus we have shown that $(u, L)$ is a solution to equation (1.1).

Uniqueness. Let $\left(v(t, x), L_{1}(d t, d x)\right)$ be another solution to the reflected SPDE (1.1) such that $E\left[\sup _{0 \leq t \leq T}\|v(t)\|^{2}\right]<\infty$ for every $T>0$. Denote by $\left\{e_{m}, m \geq 1\right\}$ the eigen basis of the Laplacian operator $\Delta=\frac{\partial^{2}}{\partial x^{2}}$ on [0,1] with Dirichlet boundary condition, namely, $\Delta e_{m}=-\lambda_{m} e_{m}$, $\lambda_{m}>0$. Define $u_{m}(t)=<u(t), e_{m}>$ and $v_{m}(t)=<v(t), e_{m}>$. Set $L^{m}(d s)=\int_{0}^{1} e_{m}(x) L(d s, d x)$ and $L_{1}^{m}(d s)=\int_{0}^{1} e_{m}(x) L_{1}(d s, d x)$. Applying the test function $e_{m}$ to both sides of the equations satisfied by $(u, L)$ and $\left(v, L_{1}\right)$ and subtracting, we get

$$
\begin{aligned}
& u_{n}(t)-v_{n}(t) \\
= & -\lambda_{n} \int_{0}^{t}\left(u_{n}(s)-v_{n}(s)\right) d s+\sum_{k=1}^{m} \int_{0}^{t}<\sigma_{k}(u(s))-\sigma_{k}(v(s)), e_{n}>d B_{k}(s) \\
& +\int_{0}^{t}<\frac{\partial}{\partial x} g(u(s))-\frac{\partial}{\partial x} g(v(t)), e_{n}>d s+\int_{0}^{t} L^{n}(d s)-\int_{0}^{t} L_{1}^{n}(d s) .
\end{aligned}
$$

For $\lambda>0$, set $h(t)=\exp \left\{-\lambda \int_{0}^{t}\|u(s)\|^{2} d s-\lambda \int_{0}^{t}\|v(s)\|^{2} d s\right\}$. Applying Ito's formula to real-valued semimartingales, it follows that

$$
\begin{aligned}
& \left|u_{n}(t)-v_{n}(t)\right|^{2} h(t) \\
= & -\lambda \int_{0}^{t} h(s)\left(\|u(s)\|^{2}+|| v(s) \|^{2}\right)\left|u_{n}(s)-v_{n}(s)\right|^{2} d s-2 \lambda_{n} \int_{0}^{t} h(s)\left|u_{n}(s)-v_{n}(s)\right|^{2} d s \\
& +2 \sum_{k=1}^{m} \int_{0}^{t} h(s)\left(u_{n}(s)-v_{n}(s)\right)<\sigma_{k}(u(s))-\sigma_{k}(v(s)), e_{n}>d B_{k}(s) \\
& +2 \int_{0}^{t} h(s)<\frac{\partial}{\partial x} g(u(s))-\frac{\partial}{\partial x} g(v(s)),\left(u_{n}(s)-v_{n}(s)\right) e_{n}>d s \\
& +\sum_{k=1}^{m} \int_{0}^{t} h(s)\left|<\sigma_{k}(u(s))-\sigma_{k}(v(s)), e_{n}>\right|^{2} d s \\
& +2 \int_{0}^{t} h(s)\left(u_{n}(s)-v_{n}(s)\right) L^{n}(d s)-2 \int_{0}^{t} h(s)\left(u_{n}(s)-v_{n}(s)\right) L_{1}^{n}(d s) .
\end{aligned}
$$


Adding up from 1 to $N$ yields

$$
\begin{aligned}
& \sum_{n=1}^{N}\left|u_{n}(t)-v_{n}(t)\right|^{2} h(t) \\
= & -\lambda \sum_{n=1}^{N} \int_{0}^{t} h(s)\left(\|\left. u(s)\right|^{2}+|| v(s)||^{2}\right)\left|u_{n}(s)-v_{n}(s)\right|^{2} d s-2 \sum_{n=1}^{N} \lambda_{n} \int_{0}^{t} h(s)\left|u_{n}(s)-v_{n}(s)\right|^{2} d s \\
& +2 \sum_{k=1}^{m} \sum_{n=1}^{N} \int_{0}^{t} h(s)<\sigma_{k}(u(s))-\sigma_{k}(v(s)),\left(u_{n}(s)-v_{n}(s)\right) e_{n}>d B_{k}(s) \\
& +2 \sum_{n=1}^{N} \int_{0}^{t} h(s)<\frac{\partial}{\partial x} g(u(s))-\frac{\partial}{\partial x} g(v(s)),\left(u_{n}(s)-v_{n}(s)\right) e_{n}>d s \\
& +\sum_{k=1}^{m} \sum_{n=1}^{N} \int_{0}^{t} h(s)\left|<\sigma_{k}(u(s))-\sigma_{k}(v(s)), e_{n}>\right|^{2} d s \\
& +2 \sum_{n=1}^{N} \int_{0}^{t} h(s)\left(u_{n}(s)-v_{n}(s)\right) L^{n}(d s)-2 \sum_{n=1}^{N} \int_{0}^{t} h(s)\left(u_{n}(s)-v_{n}(s)\right) L_{1}^{n}(d s) .
\end{aligned}
$$

We will let $N$ tend to $\infty$ to establish a bound for $|u(t)-v(t)|_{H}^{2} h(t)$. We first show that

$$
\begin{aligned}
& \lim _{N \rightarrow \infty} \sum_{n=1}^{N} \int_{0}^{t} h(s)\left(u_{n}(s)-v_{n}(s)\right) L^{n}(d s)=\int_{0}^{t} \int_{0}^{1} h(s)(u(s, x)-v(s, x)) L(d s, d x), \\
& \lim _{N \rightarrow \infty} \sum_{n=1}^{N} \int_{0}^{t} h(s)\left(u_{n}(s)-v_{n}(s)\right) L_{1}^{n}(d s)=\int_{0}^{t} \int_{0}^{1} h(s)(u(s, x)-v(s, x)) L_{1}(d s, d x) .
\end{aligned}
$$

Because of the similarity, we only prove (3.23). Note that

$$
\sum_{n=1}^{N} \int_{0}^{t} h(s)\left(u_{n}(s)-v_{n}(s)\right) L^{n}(d s)=\int_{0}^{t} \int_{0}^{1} h(s) \sum_{n=1}^{N}<u(s)-v(s), e_{n}>e_{n}(x) L(d s, d x)
$$

and

$$
F^{n}(s, x):=\sum_{n=1}^{N}<u(s)-v(s), e_{n}>e_{n}(x) \rightarrow u(s)-v(s) \quad \text { in } \quad V
$$

as $N \rightarrow \infty$. By the Sobolev embedding,

$$
\sup _{0 \leq s \leq t}\left|F^{N}(s, \cdot)\right|_{L^{\infty}} \leq C \sup _{0 \leq s \leq t}\left\|F^{N}(s, \cdot)\right\| \leq C \sup _{0 \leq s \leq t}\|u(s)-v(s)\| \in L^{2}(\Omega)
$$

As $E\left[\operatorname{Var}(L)^{2}([0, t] \times[0,1])\right]<\infty,(3.23)$ follows from the dominated convergence theorem. As $v(t, x), u(t, x)$ are nonegative, it follows from the definition of the solution that

$$
\int_{0}^{t} \int_{0}^{1} h(s)(u(s, x)-v(s, x)) L(d s, d x) \leq 0, \quad \int_{0}^{t} \int_{0}^{1} h(s)(u(s, x)-v(s, x)) L_{1}(d s, d x) \geq 0 .
$$


Let $N \rightarrow \infty$ in (3.22) to obtain

$$
\begin{aligned}
& |u(t)-v(t)|^{2} h(t) \\
\leq & -\lambda \int_{0}^{t} h(s)\left(\|u(s)\|^{2}+\|v(s)\|^{2}\right)|u(s)-v(s)|^{2} d s \\
& -2 \int_{0}^{t} h(s)\|u(s)-v(s)\|^{2} d s+2 \sum_{k=1}^{m} \int_{0}^{t} h(s)<u(s)-v(s), \sigma_{k}(u(s))-\sigma_{k}(v(s))>d B_{k}(s) \\
& +2 \int_{0}^{t} h(s)<u(s)-v(s), \frac{\partial g(u(s))-g(v(s))}{\partial x}>d s+\sum_{k=1}^{m} \int_{0}^{t} h(s)\left|\sigma_{k}(u(s))-\sigma_{k}(v(s))\right|^{2} d s .
\end{aligned}
$$

Now,

$$
\begin{aligned}
& 2 \int_{0}^{t} h(s)<u(s)-v(s), \frac{\partial g(u(s))-g(v(s))}{\partial x}>d s \\
= & -2 \int_{0}^{t} h(s)<\frac{\partial}{\partial x}(u(s)-v(s)), g(u(s))-g(v(s))>d s \\
\leq & \int_{0}^{t} h(s)\|u(s)-v(s)\|^{2} d s+C \int_{0}^{t} h(s) d s \int_{0}^{1}|g(u(s, x))-g(v(s, x))|^{2} d x \\
\leq & \int_{0}^{t} h(s)\|u(s)-v(s)\|^{2} d s+C \int_{0}^{t} h(s) d s \int_{0}^{1}\left(1+|u(s, x)|^{2}+|v(s, x)|^{2}\right)|u(s, x)-v(s, x)|^{2} d x \\
\leq & \int_{0}^{t} h(s)\|u(s)-v(s)\|^{2} d s+C \int_{0}^{t} h(s) d s\left(1+\|u(s)\|^{2}+\|v(s)\|^{2}\right)|u(s)-v(s)|_{H}^{2} .
\end{aligned}
$$

Substituting (3.27) into (3.26), choosing $\lambda$ sufficiently large and using the Lipschitz continuity of $\sigma_{k}$ we obtain

$$
\begin{aligned}
& |u(t)-v(t)|^{2} h(t) \\
\leq & 2 \sum_{k=1}^{m} \int_{0}^{t} h(s)<u(s)-v(s), \sigma_{k}(u(s))-\sigma_{k}(v(s))>d B_{k}(s) \\
& +C \int_{0}^{t} h(s)|u(s)-v(s)|_{H}^{2} d s .
\end{aligned}
$$

Taking expectation in the above inequality and applying Gronwall's inequality, it follows that $E\left[|u(t)-v(t)|^{2} h(t)\right]=0$ proving the uniqueness. The energy equation (3.12) can be proved through finite dimensional approximations as done for the uniqueness.

\section{Generalized Solutions}

In this section we will show that there exists a generalized solution for the reflected stochastic Burgers type equation when the initial function $u_{0}$ is merely in $L^{2}([0,1])$. Recall that $\left\{e_{k}, k \geq 1\right\}$ is the orthonormal basis of $H$ consisting of eigen-functions of the Laplacian operator $\Delta$ on $[0,1]$ with Dirichlet boundary condition.

Definition 4.1. We say that $(u, L)$ is a generalized solution to equation (1.1) if

(i) $u$ is an adapted $H$-valued process such that $u \in C([0, T], H) \cap L^{2}([0, T], V)$ a.s., and $L(d s, d x)$ is an adapted random measure on $[0, \infty) \times[0,1]$, 
(ii) for any $\phi \in C_{0}^{2}((0, \infty) \times[0,1] \rightarrow R)$,

$$
\begin{aligned}
& \int_{0}^{t} \int_{0}^{1} \phi(s, x) L(d s, d x) \\
= & <u(t), \phi(t)>-<u(0), \phi(0)>-\int_{0}^{t}<\frac{\partial \phi(s)}{\partial s}, u(s)>d s \\
& -\int_{0}^{t} d s<\frac{\partial^{2} \phi(s)}{\partial x^{2}}, u(s)> \\
& -\sum_{k=1}^{m} \int_{0}^{t}<\phi(s), \sigma_{k}(u(s))>d B_{k}(s)+\int_{0}^{t}<\frac{\partial \phi(s)}{\partial x}, g(u(s))>d s, a . s .,
\end{aligned}
$$

(iii) $\lim _{n \rightarrow \infty} \int_{0}^{T} \int_{0}^{1} \sum_{k=1}^{n}<u(s), e_{k}>e_{k}(x) L(d s, d x)=0$.

Theorem 4.1. Suppose (A.1) and (A.2) hold. Let $u_{0} \in H$ with $u_{0} \geq 0$. Then the reflected stochastic Burgers type equation admits a generalized solution $(u, L)$ that satisfies

$$
E\left[\sup _{0 \leq t \leq T}|u(t)|_{H}^{2}\right]+E\left[\int_{0}^{T}\|u(t)\|_{V}^{2} d t\right]<\infty
$$

for $T>0$.

Proof. Without loss of generality, we fix the time interval $[0, T]$. Take a sequence $u_{0}^{n}$ of nonnegative functions in $V$ that converges to $u_{0}$ in $H$. Let $\left(u^{n}, L^{n}\right)$ denote the unique solution of equation (1.1) with $u_{0}$ replaced by $u_{0}^{n}$. We will show that the sequence $\left(u^{n}, L^{n}\right)$ converges to a generalized solution of the equation (1.1). By energy equation proved in Theorem 3.1,

$$
\begin{aligned}
\left|u^{n}(t)\right|_{H}^{2} & =\left|u_{0}^{n}\right|_{H}^{2}+2 \int_{0}^{t} d s \int_{0}^{1}\left(u^{n}(s, x), \frac{\partial^{2} u^{n}(s, x)}{\partial x^{2}}\right) d x \\
& +2 \sum_{k=1}^{m} \int_{0}^{t}<u^{n}(s), \sigma_{k}\left(u^{n}(s)\right)>d B_{k}(s)+\sum_{k=1}^{m} \int_{0}^{t}\left|\sigma_{k}\left(u^{n}(s)\right)\right|_{H}^{2} d s .
\end{aligned}
$$

Using the above equatioin, as in the proof of Lemma 2.1 we can show that

$$
\sup _{n}\left\{E\left[\sup _{0 \leq t \leq T}\left|u^{n}(t)\right|_{H}^{2}\right]+E\left[\int_{0}^{T}\left\|u^{n}(t)\right\|^{2} d t\right]\right\} \leq C \sup _{n} E\left[\left|u_{0}^{n}\right|_{H}^{2}\right]<\infty
$$

for some constant $K$, independent of $n$. As $u_{0}^{n} \in V, u_{0}^{m} \in V$, through finite dimensional approximations as in the proof of uniqueness of Theorem 3.1 the following energy equation holds

$$
\begin{aligned}
& \left|u^{n}(t)-u^{m}(t)\right|_{H}^{2} \\
= & \left|u_{0}^{n}-u_{0}^{m}\right|_{H}^{2}-2 \int_{0}^{t} \| u^{n}(s)-\left.u^{m}(s)\right|^{2} d s \\
+ & 2 \sum_{k=1}^{m} \int_{0}^{t}<u^{n}(s)-u^{m}(s), \sigma_{k}\left(u^{n}(s)\right)-\sigma_{k}\left(u^{m}(s)\right)>d B_{k}(s) \\
+ & 2 \int_{0}^{t}<u^{n}(s)-u^{m}(s), \frac{\partial g\left(u^{n}(s)\right)}{\partial x}-\frac{\partial g\left(u^{m}(s)\right)}{\partial x}>d s \\
+ & 2 \int_{0}^{t} \int_{0}^{1}\left(u^{n}(s, x)-u^{m}(s, x)\right) L^{n}(d s, d x)-2 \int_{0}^{t} \int_{0}^{1}\left(u^{n}(s, x)-u^{m}(s, x)\right) L^{m}(d s, d x) \\
+ & \sum_{k=1}^{m} \int_{0}^{t}\left|\sigma_{k}\left(u^{n}(s)\right)-\sigma_{k}\left(u^{m}(s)\right)\right|_{H}^{2} d s .
\end{aligned}
$$


Set

$$
e_{n, m}(t)=\exp \left\{-\lambda \int_{0}^{t}\left\|u^{n}(s)\right\|^{2} d s-\lambda \int_{0}^{t}\left\|u^{m}(s)\right\|^{2} d s\right\} .
$$

Applying the chain rule, we have

$$
\begin{aligned}
& \left|u^{n}(t)-u^{m}(t)\right|_{H}^{2} e_{n, m}(t) \\
= & -\lambda \int_{0}^{t} e_{n, m}(s)\left(\left\|u^{n}(s)\right\|^{2}+\left\|u^{m}(s)\right\|^{2}\right)\left|u^{n}(s)-u^{m}(s)\right|_{H}^{2} d s \\
+ & \left|u_{0}^{n}-u_{0}^{m}\right|_{H}^{2}-2 \int_{0}^{t} e_{n, m}(s) \| u^{n}(s)-\left.u^{m}(s)\right|^{2} d s \\
+ & 2 \sum_{k=1}^{m} \int_{0}^{t} e_{n, m}(s)<u^{n}(s)-u^{m}(s), \sigma_{k}\left(u^{n}(s)\right)-\sigma_{k}\left(u^{m}(s)\right)>d B_{k}(s) \\
+ & 2 \int_{0}^{t} e_{n, m}(s)<u^{n}(s)-u^{m}(s), \frac{\partial g\left(u^{n}(s)\right)}{\partial x}-\frac{\partial g\left(u^{m}(s)\right)}{\partial x}>d s \\
+ & 2 \int_{0}^{t} \int_{0}^{1} e_{n, m}(s)\left(u^{n}(s, x)-u^{m}(s, x)\right) L^{n}(d s, d x)-2 \int_{0}^{t} \int_{0}^{1} e_{n, m}(s)\left(u^{n}(s, x)-u^{m}(s, x)\right) L^{m}(d s, d x) \\
+ & \sum_{k=1}^{m} \int_{0}^{t} e_{n, m}(s)\left|\sigma_{k}\left(u^{n}(s)\right)-\sigma_{k}\left(u^{m}(s)\right)\right|_{H}^{2} d s .
\end{aligned}
$$

From the definition of the solutions we have

$$
\begin{gathered}
2 \int_{0}^{t} \int_{0}^{1} e_{n, m}(s)\left(u^{n}(s, x)-u^{m}(s, x)\right) L^{n}(d s, d x) \leq 0 \\
-2 \int_{0}^{t} \int_{0}^{1} e_{n, m}(s)\left(u^{n}(s, x)-u^{m}(s, x)\right) L^{m}(d s, d x) \geq 0 .
\end{gathered}
$$

Following the similar arguments as in the proof of Lemma 3.1 and using (4.6), it can be proved that there exists a constant $\lambda>0$ such that

$$
\begin{aligned}
& \lim _{n, m \rightarrow \infty}\left\{E\left[\sup _{0 \leq t \leq T} \exp \left\{-\lambda \int_{0}^{t}\left(\left\|u^{n}(s)\right\|^{2}+\left\|u^{m}(s)\right\|^{2}\right) d s\right\}\left|u^{n}(t)-u^{m}(t)\right|_{H}^{2}\right]\right. \\
& \left.+E\left[\int_{0}^{T} \exp \left\{-\lambda \int_{0}^{t}\left(\left\|u^{n}(s)\right\|^{2}+\left\|u^{m}(s)\right\|^{2}\right) d s\right\}\left\|u^{n}(t)-u^{m}(t)\right\|^{2} d t\right]\right\}=0 .
\end{aligned}
$$

Thus, as in Lemma 3.2, we can deduce from (4.7) and (4.4) that $u^{n}$ converges also in probability to some process $u$ in the space $C([0, T] \rightarrow H) \cap L^{2}([0, T] \rightarrow V)$. Moreover, for any $T>0$,

$$
E\left[\sup _{0 \leq t \leq T}|u(t)|_{H}^{2}\right]+E\left[\int_{0}^{T}\|u(t)\|^{2} d t\right]<\infty .
$$

Take any $\phi \in C_{0}^{2}((0, \infty) \times[0,1] \rightarrow R)$ and use the chain rule to get

$$
\begin{aligned}
& \int_{0}^{t} \int_{0}^{1} \phi(s, x) L^{(n)}(d s, d x) \\
= & <u^{n}(t), \phi(t)>-<u_{0}^{n}, \phi(0)>-\int_{0}^{t}<\frac{\partial \phi(s)}{\partial s}, u^{n}(s)>d s \\
& -\int_{0}^{t}<\frac{\partial^{2} \phi(s)}{\partial x^{2}}, u^{n}(s)>d s \\
& -\sum_{k=1}^{m} \int_{0}^{t}<\phi(s), \sigma_{k}\left(u^{n}(s)\right)>d B_{k}(s)+\int_{0}^{t}<\frac{\partial \phi(s)}{\partial x}, g\left(u^{n}(s)\right)>d s .
\end{aligned}
$$


Since every term on the right side converges, letting $n \rightarrow \infty$ we see that $L^{n}(d s, d x)$ converges as distributions to a non-negative random measure $L(d s, d x)$ and

$$
\begin{aligned}
& \int_{0}^{t} \int_{0}^{1} \phi(s, x) L(d s, d x) \\
= & <u(t), \phi(t)>-<u_{0}, \phi(0)>-\int_{0}^{t}<\frac{\partial \phi(s)}{\partial s}, u(s)>d s \\
& -\int_{0}^{t} d s<\frac{\partial^{2} \phi(s)}{\partial x^{2}}, u(s)> \\
& -\sum_{k=1}^{m} \int_{0}^{t} d s<\phi(s), \sigma_{k}(u(s))>d B_{k}(s)+\int_{0}^{t} d s<\frac{\partial \phi(s)}{\partial x}, g(u(s))>d s .
\end{aligned}
$$

To show $(u, L)$ is a generalized solution, it remains to show that

$$
\lim _{n \rightarrow \infty} \int_{0}^{T} \int_{0}^{1} \sum_{j=1}^{n}<u(t), e_{j}>e_{j}(x) L(d s, d x)=0 .
$$

Let $u_{j}(t)=<u(t), e_{j}>$ and $L^{j}(d s)=\int_{0}^{1} e_{j}(x) L(d s, d x)$. Choose $\phi(t, x)=e_{j}$ in (4.10) to get

$$
\begin{aligned}
u_{j}(t)= & -\lambda_{j} \int_{0}^{t} u_{j}(s) d s+\sum_{k=1}^{m} \int_{0}^{t}<\sigma_{k}(u(s)), e_{j}>d B_{k}(s) \\
& +\int_{0}^{t}<\frac{\partial}{\partial x} g(u(s)), e_{j}>d s+\int_{0}^{t} L^{j}(d s) .
\end{aligned}
$$

By Ito's formula for real-valued semimartingales, it follows that

$$
\begin{aligned}
\left|u_{j}(t)\right|^{2} & =\left|u_{j}(0)\right|^{2}-2 \lambda_{j} \int_{0}^{t}\left|u_{j}(s)\right|^{2} d s+2 \int_{0}^{t} u_{j}(s) L^{j}(d s) \\
& +2 \sum_{k=1}^{m} \int_{0}^{t} u_{j}(s)<\sigma_{k}(u(s)), e_{j}>d B_{k}(s) \\
& +2 \int_{0}^{t} u_{j}(s)<\frac{\partial}{\partial x} g(u(s)), e_{j}>d s \\
& +\sum_{k=1}^{m} \int_{0}^{t}\left|<\sigma_{k}(u(s))-\sigma_{k}(v(s)), e_{j}>\right|^{2} d s
\end{aligned}
$$

Take the sum from 1 to $n$ to obtain

$$
\begin{aligned}
& \sum_{j=1}^{n}\left|u_{j}(t)\right|^{2} \\
= & \sum_{j=1}^{n}\left|u_{j}(0)\right|^{2}-2 \sum_{j=1}^{n} \lambda_{j} \int_{0}^{t}\left|u_{j}(s)\right|^{2} d s+2 \int_{0}^{t} \int_{0}^{1} \sum_{j=1}^{n} u_{j}(s) e_{j}(x) L(d s, d x) \\
& +2 \sum_{k=1}^{m} \int_{0}^{t}<\sigma_{k}(u(s)), \sum_{j=1}^{n} u_{j}(s) e_{j}>d B_{k}(s) \\
& +2 \int_{0}^{t}<\frac{\partial}{\partial x} g(u(s)), \sum_{j=1}^{n} u_{j}(s) e_{j}>d s+\sum_{k=1}^{m} \sum_{j=1}^{n} \int_{0}^{t}\left|<\sigma_{k}(u(s)), e_{j}>\right|^{2} d s .
\end{aligned}
$$


For $f \in V$, recall that

$$
\|f\|^{2}=\sum_{j=1}^{\infty} \lambda_{j}<f, e_{j}>^{2} .
$$

For $u \in V$, put $B(u)=\frac{\partial}{\partial x} g(u(x))$. We have the following estimate.

$$
\|B(u)\|_{V^{*}} \leq C\left(|u|_{H}^{\frac{3}{2}}\|u\|_{V}^{\frac{1}{2}}+1\right) .
$$

Let us prove (4.16). Recall that $V=D\left(A^{\frac{1}{2}}\right)$, where $-A$ is the Laplacian operator on $[0,1]$ with the Dirichlet boundary condition. Note that $\bar{e}_{k}=\frac{1}{\sqrt{\lambda_{k}}} e_{k}, k \geq 1$ forms an orthonormal basis of $V$. Recall that any element $l \in V^{*}$ can be identified through the Riesz representation theorem as an element $\bar{l}$ in $V$ and moreover,

$$
\|l\|_{V^{*}}^{2}=\|\bar{l}\| \|_{V}^{2}=\sum_{k=1}^{\infty}<\bar{l}, \bar{e}_{k}>^{2}=\sum_{k=1}^{\infty}\left(l\left(\bar{e}_{k}\right)\right)^{2} .
$$

Keeping the assumption of $g$ in mind we have

$$
\begin{aligned}
\|B(u)\|_{V^{*}}^{2} & =\sum_{k=1}^{\infty}\left(B(u)\left(\bar{e}_{k}\right)\right)^{2} \\
& =\sum_{k=1}^{\infty}\left(\frac{1}{2} \frac{1}{\sqrt{\lambda_{k}}} \int_{0}^{1} \frac{\partial}{\partial \xi}[g(u(\xi))] e_{k}(\xi) d \xi\right)^{2} \\
& =\sum_{k=1}^{\infty}\left(\frac{1}{2} \frac{1}{\sqrt{\lambda_{k}}} \int_{0}^{1} g(u(\xi)) \frac{\partial}{\partial \xi} e_{k}(\xi) d \xi\right)^{2} \\
& =\sum_{k=1}^{\infty}\left(\frac{1}{2} \int_{0}^{1} g(u(\xi)) \sqrt{2} \cos (k \pi \xi) d \xi\right)^{2} \\
& \leq C \int_{0}^{1} g(u(\xi))^{2} d \xi \leq C\left(|u|_{L^{4}}^{4}+1\right)
\end{aligned}
$$

where we have used the fact that $\{\sqrt{2} \cos (k \pi \xi) ; k \geq 1\}$ also forms an orthonormal system of $L^{2}(0,1)$. By the Sobolev embedding theorem (see, e.g. Theorem 6, Chapter 5 in $[E]$ ), we have

$$
H^{\frac{1}{4}}(0,1) \subset L^{4}(0,1)
$$

where $H^{\frac{1}{4}}(0,1)$ is the usual Sobolev space of order $\frac{1}{4}$. Using the following well known interpolation inequality (see e.g. Section 4.3 in $[\mathrm{T}]$ )

$$
|u|_{L^{4}}^{4} \leq C|u|_{H}^{3}\|u\|_{V}
$$

(4.16) follows from (4.17) and (4.18). To pass to the limit in (4.14) we further notice that

$$
\sum_{j=1}^{n} u_{j}(s) e_{j} \rightarrow u \quad \text { in } \quad L^{2}([0, T] \rightarrow V),
$$

and

$$
\int_{0}^{T}\|B(u(t))\|_{V^{*}}^{2} d t \leq C\left(\sup _{0 \leq t \leq T}|u(t)|_{H}^{3}+1\right) \int_{0}^{T}\|u(t)\|^{2} d t<\infty
$$


Now we let $n \rightarrow \infty$ in (4.14) to get that

$$
\begin{aligned}
|u(t)|^{2}= & |u(0)|^{2}-2 \int_{0}^{t}\|u(s)\|^{2} d s+2 \lim _{n \rightarrow \infty} \int_{0}^{t} \int_{0}^{1} \sum_{j=1}^{n} u_{j}(s) e_{j}(x) L(d s, d x) \\
& +2 \sum_{k=1}^{m} \int_{0}^{t}<\sigma_{k}(u(s)), u(s)>d B_{k}(s)+\sum_{k=1}^{m} \int_{0}^{t}\left|\sigma_{k}(u(s))\right|^{2} d s,
\end{aligned}
$$

where we have used the fact that

$$
\int_{0}^{t}<\frac{\partial}{\partial x} g(u(s)), u(s)>d s=0
$$

On the other hand, since $u^{n}$ converges in probability to $u$ in the space $C([0, T] \rightarrow H) \cap L^{2}([0, T] \rightarrow$ $V$ ), we let $n \rightarrow \infty$ in (4.3) to obtain

$$
\begin{aligned}
|u(t)|^{2}= & |u(0)|^{2}-2 \int_{0}^{t}\|u(s)\|^{2} d s \\
& +2 \sum_{k=1}^{m} \int_{0}^{t}<\sigma_{k}(u(s)), u(s)>d B_{k}(s)+\sum_{k=1}^{m} \int_{0}^{t}\left|\sigma_{k}(u(s))\right|^{2} d s .
\end{aligned}
$$

Comparing (4.19) with (4.20) we conclude that

$$
\lim _{n \rightarrow \infty} \int_{0}^{t} \int_{0}^{1} \sum_{j=1}^{n} u_{j}(s) e_{j}(x) L(d s, d x)=0
$$

completing the proof of the theorem.

Acknowledgement. I thank the referee for the very careful reading and useful suggestions.

\section{References}

[DZ] G. Da Prato and J. Zabczyk: Stochastic Equations in Infinite Dimensions. Cambridge University Press, 1992.

[DMZ] R. C. Dalang, C. Mueller, L. Zambotti: Hitting properties of parabolic SPDE's with reflection. Ann. Probab. 34(4)(2006), 1423-1450.

[DP1] C. Donai-Martin, E. Pardoux: White noise driven SPDEs with reflection. Probab. Theory Relat. Fields 95, 1-24(1993).

[DP2] C. Donai-Martin, E. Pardoux: EDPS réfléchies et calcul de Malliavin. (French)[SPDEs with reflection and Malliavin Calculus]. Bull. Sci. Math. 121(5)(1997), 405-422.

[DZ] A. Debussche, L. Zambotti: Conservative stochastic Cahn-Hilliard equation with reflection. Annals of Probability 35(5) (2007) 1706-1739.

[E] L. C. Evans: Partial Differential Equations. American Mathematical Society 1998.

[FO] T. Funaki, S. Olla: Fluctuations for $\nabla \phi$ interface model on a wall. Stochastic Process. Appl. 94(1)(2001), 1-27.

[GP] A. Gegout-Petit, E. Pardoux: Equations Différentielles Stochastiques Rétrogrades Réfléchies Dans Un Convexe. Stochastics and Stochastics Reports 57 (1996) 111-128. 
[GN] I. Gyöngy and D. Nualart: On the stochastic Burgers' equations in the real line. Annals of Probability 27:2 (1999) 782-802.

[HP] U. G. Haussmann, E. Pardoux: Stochastic variational inequalities of parabolic type. App. Math. Optim. 20(1989), 163-192.

[NP] D. Nualart, E. Pardoux: White noise driven by quaslinear SPDEs with reflection. Probab. Theory Relat. Fields 93,77-89(1992).

[P] E. Pardoux: Stochastic partial differential equations and filtering of diffusion processes. Stochastics 3(1979), 127-167.

[PR] C. Prévot and M. Röckner: A concise course on stochastic partial differential equations. Lecture Notes in Mathematics 1905, Springer Berlin 2007.

[T] H. Triebel: Interpolation Theory, Function Spaces, Differential Operators. North Holland 1978.

[XZ] T. Xu and T.Zhang: White noise driven SPDEs with reflection: existence, uniqueness and large deviation principles. Stochastic Processes and Their Applications 119:10 (2009) 34533470 .

[ZA] L. Zambotti: A reflected stochastic heat equation as symmetric dynamics with respect to the 3-d Bessel bridge. Journal of Functional Analysis 180, 195-209(2001).

[Z] T.Zhang : White noise driven SPDEs with reflection: strong Feller properties and Harnack inequalities. Potential Analysis 33:2 (2010) 137-151. 